\title{
A Modal-tense Sortal Logic with Variable-Domain Second-order Quantification
}

\author{
Max A. Freund \\ Doctoral Program in Philosophy and Graduate Program in \\ Cognitive Science \\ University of Costa Rica \\ Costa Rica
}

\begin{abstract}
We propose a new intensional semantics for modal-tense second-order languages with sortal predicates. The semantics provides a variabledomain interpretation of the second-order quantifiers. A formal logical system is characterized and proved to be sound and complete with respect to the semantics. A contemporary variant of conceptualism as a theory of universals is the philosophical background of the semantics. Justification for the variable-domain interpretation of the second-order quantifiers presupposes such a conceptualist framework.
\end{abstract}

\section{Introduction}

A notion that plays an important role in certain areas of philosophy, cognitive psychology and logic is that of a sortal predicate or sortal concept. Notwithstanding its importance, the notion is not sufficiently precise and, as a consequence of this feature, a controversy exists regarding the criteria for setting apart sortal predicates from other kind of predicates. However, there are universally accepted prototypes of linguistic expressions that count as clear cases of sortal and non-sortal predicates. Most of our common count nouns (such as "ant" and "dog") are definite cases of sortal predicates. ${ }^{1}$ Adjectives and intransitive verbs are clear examples of non-sortal predicates. On the basis of these prototypes, any procedure establishing the required distinction will have to include among the defining features the idea that sortal terms generally provide counting, identification and classification criteria. $^{2}$ This means that, if $\mathrm{S}$ is a sortal predicate, it will make sense to ask how many S's there are, whether $z$ is the same $\mathrm{S}$ as $x$, and finally whether $x$ is an $\mathrm{S}$.

The last two kinds of questions illustrate two important logical aspects of sortal predicates, namely, regarding these terms we can form 1) relative (sortal) 
identities such as "a is the same spider as b", and "a is the same horse as b", and 2) sortal predications such as "John is a man". The counting criterium, on the other hand, serves as the foundation for a third logical feature: by means of sortal predicates we can construct relative quantifiers, such as "every spider and "some horse".

Now, when a formal language allows for the formal representation of sortal quantification and identity, and is such that its logical syntax assumes such representations as undefinable, we shall speak here of a (formal) language for sortals. ${ }^{3}$ In previous papers, we have studied extensional and intensional languages for sortal predicates and developed formal semantic systems for them together with the corresponding sound and complete formal logical systems. These systems presuppose a modern form of a conceptualist theory of universals as philosophical background theory. ${ }^{4}$

In general, conceptualism as a theory of universals presupposes that general terms stand for concepts and constitute their semantic basis. ${ }^{5}$ The modern variant of conceptualism assumed in the above papers extends the general view of conceptualism to all meaningful linguistic expressions, with the exception of sentences; that is, except for sentences, every meaningful expression of a language will have a concept as its semantic ground. In addition to such an extension, the contemporary philosophical theory in question assumes the view that concepts constitute cognitive capacities or cognitive structures based on such capacities.

As in the previous papers, we shall here also adopt the above contemporary version of conceptualism. Thus, as to be expected, sortal predicates will here stand for concepts and, consequently, for certain kind of cognitive capacities or structures based on such capacities. We shall here refer to such concepts as sortal concepts.

Given the interpretation of concepts as cognitive capacities or cognitive structures based on such capacities, a variation through time of the total set of sortal concepts is to be expected. This is because such capacities or structures ontologically depend on the concrete individuals that have formed them. Since existence of such individuals is finite, the subsistence of concepts is also limited.

In addition to actual concepts, we can take into account possible concepts. Possibility in this case is to be understood in terms of natural possibility, since this is the sense of possibility more pertinent for the view of concepts assumed in this paper. Therefore, with natural possibility in view, an additional parameter for variation in the set of concepts is introduced: the set of concepts formed at one naturally possible world might be different from the set of concepts formed at another possible world. This parameter of variation together with the temporal parameter suggest a variable-domain interpretation of (second-order) quantification over sortal concepts. In accordance with this interpretation, the range of a second-order quantifier over sortal concepts, at a possible world and time interval, would be constituted by the total set of sortal concepts remaining or being developed at that same world and interval. As far as we know, in the development of the logic for sortal predicates such an interpretation has not been considered. That is, logics for sortals which include in their logical syntax second-order quantifiers over sortal concepts have assigned a constant-domain 
interpretation to such quantifiers. In the constant-domain interpretation, quantification is over the totality of sortal concepts that in principle can be formed or constructed, that is, over all the concepts that can be formed at some possible world or other.

Our goal in the present paper is to construct a logic for sortals in which second-order quantifiers over sortals are given a variable-domain interpretation. Since time and possible worlds are here involved, in order to capture the interpretation in question more faithfully, our logic will be modal-tense. In other words, the language of our logic will be a second-order sortal language with modal and tense operators. Thus, the logical syntax of the language will allow for the concatenation of universal quantifiers with sortal predicate variables and contain, in addition to expressions representing relative identity and relative quantification, also modal and tense operators together with the classical propositional connectives.

Technically speaking, we shall here formulate a set-theoretic possible world semantics for the above two dimensional second-order sortal language. The semantics will provide a variable-domain interpretation of the second-order quantifiers, that is, their range might vary across possible worlds and time-points. We shall develop a formal logical system, prove its soundness and completeness with respect to the notion of validity provided by the semantics. The semantics will assume elements of the abovementioned modern version of conceptualism as a theory of universals as its philosophical background. It will also capture the intuitive interpretations of the propositional operators.

We should note that in Freund (2007) we have constructed a tense-modal logic for sortals but with a constant-domain interpretation of the second-order quantifiers over sortal concepts. Clearly, by providing a variable-domain interpretation of the second order quantifiers, the semantics of the present article will differ from that in Freund (2007).

\section{Philosophical preliminaries: concepts, time-intervals, and possible worlds.}

As indicated, we shall here assume a modern version of conceptualism as philosophical background. In the present section, we shall present some features of this philosophical theory that are relevant for the philosophical justification of the logic here characterized. ${ }^{6}$

As a theory of universals, we have stated that conceptualism postulates concepts as the entities general terms stand for. This sort of terms includes, for example, common nouns and adjectives. Another universal important feature of conceptualism concerns predication. Predication is explained in terms of the notion of an entity falling under a concept. So, the assertion that John is a person is interpreted as John falls under the concept of being a person. Thus, for conceptualism, predication essentially depends on concepts.

It is important to note that conceptualism, like nominalism and realism, also accepts the idea that there is predication in language, that is, that we can 
attribute (monadic or relational) predicates of individuals. Nominalism, in its original version, assumes that predication in language is the only manner in which we can justifiably talk of predication. That is, according to nominalism the only sort of predication is the attribution of a predicate (or rather of a predicate token). Modern versions of nominalism interpret predication in terms of membership in a class. In the case of realism, predication is based on properties and relations. As such, predication should be understood as the instantiation of a property or a relation. It is this sort of predication that constitutes the basis and accounts for predication in language. Contrariwise to realism and nominalism, conceptualism looks at predication in language as grounded on the predication of concepts. This latter sort of predication is tantamount to the notion of an object falling under a concept.

The contemporary variant of conceptualism here assumed adopts the above two general features. In addition to these features, it extends the conceptualist view regarding general terms to all meaningful linguistic expressions, with the exception of sentences. ${ }^{7}$ So, for example, all sort of referential expressions like quantifiers phrases, definite descriptions, etc, will have to be taken as standing for concepts. Another addition to the general conceptualist view is an interpretation of concepts as cognitive capacities or structures based on such capacities. For instance, the sortal concept of being a house will be a cognitive capacity whose exercise would allow us to identify, classify and count houses. The complex predicate "to be round and red" will stand for a cognitive structure based on the concepts of being round and being red. This structure is a construction from these two concepts by means of the logical operation of conjunction. When exercised, the structure in question would allow us to classify red and round objects.

It is clear that, unlike properties (which are the universals postulated by logical realism) and classes (which are the universals according to a version of nominalism), concepts as cognitive capacities or structures are entities ontologically dependent on time-bounded individuals. These are the individuals that are causally responsible for the concepts. Such individuals contain biological systems capable of carrying out a processing of stimulae or information from the external world. This processing and their biological structure allow the individuals in question to develop cognitive capacities or structures and, in particular, concepts. These cognitive capacites and structures subsist in those individuals but since the life-span of the individuals is clearly finite, the subsistence of concepts will also be limited. In this way, time-intervals can be assigned to sets of concepts, namely: the time-intervals during which those concepts subsist in the biological individuals generating and storing them.

Now, if we take into account possible transformations in actual biological structures (i.e, so called epigenetic changes), we get another important parameter related to the formation of concepts. Such transformations might be induced by possible but not actual modifications in the bio-physical or chemical environment that might render biological systems alternative to those of the actual world. This opens up the possibility of individuals with the potential to develop concepts different from those of the actual world. In other words, transforma- 
tions in the biological structures might causally imply formation of concepts different from those of the actual world.

Additional factors that may have as a consequence construction of a different set of concepts are material conditions distinct from those of the actual world. These conditions might provide stimulae different from those actually given.

Now, we can understand the above scenarios in terms of possible worlds, if the concept of being a possible world is interpreted as in natural necessity. This interpretation is congenial with the variant of conceptualism assumed in the present paper. This is because such a version of conceptualism is a sociobiologically based theory of the capacity for systematic concept formation committed to the existence of natural properties and relations. Some of these properties and relations provide the causal ground for the laws governing conceptformation. These laws characterize the structure of the intellect as a biologically based organon.

In sum, the possible laws governing concept-formation are those determined by the causal matrix of the naturally possible worlds. Since this matrix is common to all these worlds, any of such worlds is to be viewed as a causal alternative to the others. In other words, what is causally or naturally necessary in one of such worlds is causally necessary in any of the others.

Clearly, what would differentiate one naturally possible world from the others will not be the natural properties and relations themselves inscribed in its causal matrix (since this is what is common to all of them) but rather the concrete individuals that exist in any of such worlds. The concrete individuals at a possible world are the entities that manifest at that world some of the natural properties and relations. As such, concrete individuals might exist in more than one naturally possible world but they do not have to exist in all of such worlds. The same applies to concepts, since their being depends on some of those individuals.

By above then, two dimensions can be associated to sets of concepts: the temporal dimension and the modal dimension of natural possibility. Concepts are entities that are developed by and ontologically dependent on individuals, in a time-interval or intervals at a naturally possible world or worlds. Accordingly, the set of concepts associated to a given time-interval and naturally possible world will be the set of concepts developed by and subsisting in the individuals existing at that time-interval and world. Consequently, the set of concepts subsisting at a given time-interval and possible world might differ from those at other time-intervals and/or possible worlds. This is clearly due to the possibility, among others already mentioned, that the set of individuals at one possible world and time-interval might be different from the set of individuals at another possible world and time-interval.

\section{Philosophical preliminaries: sortal, predicable, and referential concepts}


Up to now, we have focused on the development of concepts and its ontological dependence on actual and possible individuals. We want now to take into account the different sorts of concepts that such a development might yield. The difference among concepts is to be found in the role they are meant to fullfill. Primarily, we have the distinction between sortal and predicable concepts. We have already referred to the former kind of concepts. They are those sortal predicates stand for. Hence, sortal concepts are intersubjectively realizable cognitive capacities whose uses, in thought and communication, are associated with certain criteria by which we are able to distinguish, count, identify and classify objects.

Predicable concepts, on the other hand, are intersubjectively realizable cognitive capacities or structures that only enable us to classify and relate objects. Predicates such as "black", "big", "hard", "smaller" and "older" should be viewed as representing predicable concepts. In general, non-sortal monadic predicates and $n$-place predicate expressions, (for $\mathrm{n}>1$ ), also known as relational predicates, will stand for predicable concepts.

In addition to sortal and predicable concepts, we have the referential concepts, that is, those whose exercise would allow us to refer. In this paper, we shall only take into account referential concepts that are represented either by relative sortal quantifiers or by second-order quantifiers over sortal concepts. Expressions of a form logically similar to "every S" or "some S" (where S is a sortal predicate), such as "every horse" and "some houses", stand for concepts of the former kind. Thus, this sorth of concepts constitute intersubjectively realizable cognitive structures whose exercise would enable us to refer to objects distinguished, counted and classified by sortal concepts.

Referential concepts of the other kind are represented by expressions with logical content similar to "for some sortal concept" and "for every sortal concept". They are cognitive structures whose exercise allow us to refer to the sortal concepts themselves. Two interpretations of this sort of second-order reference are at hand, viz.: the counterfactual and the variable-domain interpretations.

In the case of the counterfactual interpretation, the range of quantification comprises the class of all sortal concepts that in principle can be formed or constructed. This class would contain all of the concepts that can be formed in one or another naturally possible world at a time interval. In contraposition to the counterfactual interpretation, there is the variable domain interpretation. Here the range of quantification might vary from one possible world and/or time interval to another. In this case, second-order quantification would be closely tied to time and modality, capturing in this way, more closely, the dynamics involved in the construction and the subsistence of concepts.

As noticed in the introduction, we have already developed a logic for the counterfactual interpretation in a tense-modal context. We also pointed out that, contrarily to the case for the counterfactual interpretation, a tense-modal sortal logic that assumes the variable domain interpretation as primitive has not been formulated. In what follows, we develop such a logic. In addition to the fact that this logic has not been up till now developed, the idea that the variable domain intepretation reflects more closely the above mentioned 
dynamics of concept formation motivates the work in this paper.

\section{Language and Semantics}

We now characterize the formal language of this paper. The set of its primitive logical symbols will be constituted by the expressions $\neg, \rightarrow,=, \forall,(), G,$, and $\square$. The first two symbols will stand for classical negation and material implication, the penultimate and antepenultimate symbols will formally represent the temporal operators "it will always be the case" and "it has always been the case". The last symbol will stand for the modal operator "it is naturally necessary that $\mathrm{p} "$. The classical propositional operators of conjunction, disjunction and material equivalence will be represented by the symbols \&, $\vee$ and $\leftrightarrow$, respectively, and defined in the usual way.

We shall assume denumerably many individual variables, sortal term variables and, for each positive integer $n, n$-place predicate variables. We shall use " $x$ ", " $y$ " and " $z$ " with or without numerical subscripts to refer (in the metalanguage) to individual variables and, except for " $P$ ", " $F$ ", " $G$ " and " $H$ ", upper case letters in italics to refer to sortal term variables. Atomic well formed formulas are expressions either of the form of a relative identity $\left(a={ }_{L} b\right)$, where $a$ and $b$ are individual variables and $L$ is a sortal term variable, or of the form $\pi x_{1} \ldots x_{n}$, where $\pi$ is an $n$-place predicate variable and $x_{1} \ldots x_{n}$ are individual variables. The set of well formed formulas (wffs, for short) is the smallest set containing the atomic well formed formulas and such that $\neg \varphi,(\varphi \rightarrow \delta), \forall x L \varphi, G \varphi, H \varphi$, $\square \varphi$ and $\forall L \varphi$ are in the set whenever $\varphi, \delta$ are in the set, and $L$ is a sortal term variable.

Intuitively speaking, the expressions " $x=_{L} y$ " (for any sortal term $L$ ) will formally represent sortal identity, that is, their intuitive content is that of the expression $x$ is the same $L$ as $y$. In accordance with this interpretation, any statement of the form " $x={ }_{L} y$ " will clearly entail that $x$ is an $L$, and so that $x$ falls under the sortal concept the sortal term $L$ stands for. Take, for example, the statement John $={ }_{\text {Person }}$ Peter. This clearly implies that John is a person and, consequently, that John falls under the sortal concept of being a person. For these reasons, the identity principle, "for every sortal $L, x={ }_{L} x$ " is not intuitively valid, since it would entail that any object falls under every sortal concept. These intuitive features of relative (sortal) identity will be reflected in the formal semantics, in particular, in clause 1 of the definition of truth for the formal models of this paper. As the reader will notice, the semantics validates a different identity principle, namely: $x={ }_{L} y \rightarrow x={ }_{L} x$. This principle is assumed in the formal system as an axiom (viz., A7).

Formulas of the form " $\forall x L \varphi$ ", for any given sortal term $L$, will formally represent relative sortal quantification with respect to the sortal concept $L$. As an intuitive example, let $L$ be the sortal term "dog" and $\pi$ the monadic predicate "is white", then $\forall x L \pi x$ will formally stand for "Every dog is white". Another intuitive example is the following: let $S$ be the sortal term "man" and 
$\pi$ the monadic predicate "is mortal", then " $\forall x S \pi x$ " will represent "Every man is mortal".

Finally, formulas of the form " $\forall L \varphi$ " will have to be read as "Every sortal concept is such that $\varphi$ ", where the expression "every sortal concept" has to be given a variable-domain interpretation.

Hereafter, we shall make use of lower case greek letters $\varphi, \sigma, \delta, \psi$ and $\gamma$ to refer to wffs, $\pi$ to refer to predicate variables and upper case greek letters such as $\Gamma, \Delta$ and $\Sigma$ to refer to sets of wffs. We shall generally drop the use of parentheses in a given context, if ambiguity is not possible in that context. The concepts of a bound and free occurrence of a variable are understood in the usual way. If $\alpha$ and $\beta$ are variables of the same type, then by $\varphi^{\alpha} / \beta$ is meant the wff that results by replacing each free occurrence of $\beta$ by a free occurrence of $\alpha$, if such a wff exists; otherwise $\varphi^{\alpha} / \beta$ is $\varphi$ itself. We shall say that $\alpha$ is free for $\beta$ in $\varphi$, if $\varphi^{\alpha} / \beta$ is not $\varphi$ unless $\alpha$ is $\beta$.

We now construct the semantics for the above language. We first define a frame for a variable-domain modal-tense sortal logic (VMTS-frame, for short), as a structure $\langle\mathcal{D}, \mathcal{S}, \mathcal{W}, \mathcal{T}, \mathcal{R}\rangle$, where

1. $\mathcal{D}$ is a domain of discourse, empty or otherwise,

2. $\mathcal{W}$ and $\mathcal{T}$ are non-empty sets,

3. $\mathcal{S}$ is a function from $\mathcal{W} \times \mathcal{T}$ into $\wp\left(\wp(\mathcal{D})^{\mathcal{W} \times \mathcal{T}}\right.$ ) (where $\wp(\mathcal{D})$ stands for the power set of $\mathcal{D}$ and $\wp(\mathcal{D})^{\mathcal{W} \times \mathcal{T}}$ for the set of functions from $\mathcal{W} \times \mathcal{T}$ into the power set of $\mathcal{D})$,

4. $\mathcal{R}$ is a serially ordered relation in $\mathcal{T}$, i.e., $R \subseteq \mathcal{T} \times \mathcal{T}$, and $\mathcal{R}$ is transitive, irreflexive and connected. In other words, $\mathcal{R}$ satisfies the following conditions: (i) for every $\alpha, \beta, \gamma \in \mathcal{T}$, if $\alpha \mathcal{R} \beta$ and $\beta \mathcal{R} \gamma$, then $\alpha \mathcal{R} \gamma$; (ii) for every $\alpha, \beta, \in \mathcal{T}$, either $\alpha=\beta$ or $\alpha \mathcal{R} \beta$ or $\beta \mathcal{R} \alpha$; and for every $\alpha \in \mathcal{T}$, it is not the case that $\alpha \mathcal{R} \alpha$.

We should note that $\mathcal{D}$ represents the set of concrete individuals existing at some possible world or other, $\mathcal{W}$ the set of naturally possible worlds, $\mathcal{T}$ the set of time-points, and $\mathcal{R}$ the earlier-than relation.

For each $t$ and $w, \mathcal{S}(t, w)$ stands for the set of sortal concepts that have been formed at possible world $w$ and time $t$ (and maybe previously to $t$ ). When a given function $f$ is a member of $\mathcal{S}(t, w)$ (that is, when $f$ set-theoretically represents a sortal concept $C), f$ is to be intuitively understood as assigning to each possible world $j$ and time-point $k$ the set of objects existing at $j$ and $k$ that fall under the sortal concept $C$. This corresponds to the way sortal concepts are viewed within the philosophical framework of this paper, that is, as cognitive capacities providing identity criteria only for things that exist .

Regarding the members of $T$ (viz., the time-points), we should note that a common sense view is here presupposed according to which they are serially ordered by the earlier-than relation. Clause 4 above gathers this common sense view.

Australasian Journal of Logic (12:1) 2014, Article no. 5 
By an assignment (of values to variables) in an VMTS-frame $<\mathcal{D}, \mathcal{S}, \mathcal{W}, \mathcal{T}$, $\mathcal{R}>$, we shall understand a function $\mathcal{A}$ with the set of variables (of all types) as domain and such that (i) $\mathcal{A}(x) \in \mathcal{D}$, for each individual variable $x$, (ii) $\mathcal{A}(L) \in \bigcup_{(i, j) \in \mathcal{W} \times \mathcal{T}} \mathcal{S}(i, j)$, for each sortal term variable $L$, and (iii) for each positive integer $n$ and $n$-place predicate variable $\pi, \mathcal{A}(\pi) \in \wp(\mathcal{D})^{\mathcal{W} \times \mathcal{T}}$. Clause (ii) expresses the idea that there are no sortal concepts other than those that have been formed by individuals at a possible world or other.

By a variable-domain modal-tense sortal model ( VMTS-model, for short) we shall mean an ordered pair $\mathfrak{A}=\langle<\mathcal{D}, \mathcal{S}, \mathcal{W}, \mathcal{T}, \mathcal{R}\rangle, \mathcal{A}\rangle$, where $\mathcal{A}$ is an assignment in the VMTS-frame $<\mathcal{D}, \mathcal{S}, \mathcal{W}, \mathcal{T}, \mathcal{R}>$. If $\mathfrak{A}$ is $<<\mathcal{D}, \mathcal{S}, \mathcal{W}, \mathcal{T}$, $\mathcal{R}>, \mathcal{A}>$, by $\mathfrak{A}(d / a)$ should be understood the ordered pair $<<\mathcal{D}, \mathcal{S}, \mathcal{W}, \mathcal{T}$, $\mathcal{R}>, \mathcal{A}(d, a)>$, where $\mathcal{A}(d, a)$ is like $\mathcal{A}$ except for assigning $d$ to $a$, and $a$ is either an individual or sortal term variable.

Let $\mathfrak{A}$ be a VMTS-model $\langle<\mathcal{D}, \mathcal{S}, \mathcal{W}, \mathcal{T}, \mathcal{R}\rangle, \mathcal{A}\rangle$. Where $i \in \mathcal{W}, j \in \mathcal{T}$, we shall define the truth-value of $\varphi$ in $\mathfrak{A}$ at $i$ and $j$ (in symbols, $\operatorname{Val}(\varphi, \mathfrak{A}, i, j)$ ) as follows:

1. $\operatorname{Val}\left(x=_{L} y, \mathfrak{A}, i, j\right)=1$ if $\mathcal{A}(x)=\mathcal{A}(y)$ and $\mathcal{A}(y) \in \mathcal{A}(L)(i, j)$; otherwise $\operatorname{Val}\left(x=_{S} y, \mathfrak{A}, i, j\right)=0$.

2. $\operatorname{Val}\left(\pi x_{1} \ldots x_{n}, \mathfrak{A}, i, j\right)=1$ if $\left\langle\mathcal{A}\left(x_{1}\right), \ldots, \mathcal{A}\left(x_{n}\right)>\in \mathcal{A}(\pi)(i, j)\right.$; otherwise $\operatorname{Val}\left(\pi x_{1} \ldots x_{n}, \mathfrak{A}, i, j\right)=0$.

3. $\operatorname{Val}(\neg \varphi, \mathfrak{A}, i, j)=1$ if $\operatorname{Val}(\varphi, \mathfrak{A}, i, j) \neq 1$; otherwise $\operatorname{Val}(\neg \varphi, \mathfrak{A}, i, j)=0$.

4. $\operatorname{Val}(\varphi \rightarrow \gamma, \mathfrak{A}, i, j)=1$ if $\operatorname{Val}(\neg \varphi, \mathfrak{A}, i, j)=1$ or $\operatorname{Val}(\gamma, \mathfrak{A}, i, j)=1$; otherwise $\operatorname{Val}(\varphi \rightarrow \gamma, \mathfrak{A}, i, j)=0$

5. $\operatorname{Val}(\forall L \varphi, \mathfrak{A}, i, j)=1$ if for every $d \in \mathcal{S}(i, j), \operatorname{Val}(\varphi, \mathfrak{A}(d / L), i, j)=1$; otherwise $\operatorname{Val}(\forall L \varphi, \mathfrak{A}, i, j)=0$.

6. $\operatorname{Val}(\forall x L \varphi, \mathfrak{A}, i, j)=1$ if for every $d \in \mathcal{A}(L)(i, j), \operatorname{Val}(\varphi, \mathfrak{A}(d / x), i, j)=1$; otherwise $\operatorname{Val}(\forall x L \varphi, \mathfrak{A}, i, j)=0$.

7. $\operatorname{Val}(\square \varphi, \mathfrak{A}, i, j)=1$ if for every $k \in \mathcal{W}, \operatorname{Val}(\varphi, \mathfrak{A}, k, j)=1$; otherwise $\operatorname{Val}(\square \varphi, \mathfrak{A}, i, j)=0$.

8. $\operatorname{Val}(G \varphi, \mathfrak{A}, i, j)=1$ if for every $k \in \mathcal{T}$, if $j R k, \operatorname{Val}(\varphi, \mathfrak{A}, \mathfrak{i}, k)=$ 1 ;otherwise $\operatorname{Val}(G \varphi, \mathfrak{A}, i, j)=0$.

9. $\operatorname{Val}(H \varphi, \mathfrak{A}, i, j)=1$ if for every $k \in \mathcal{T}$, if $k R j, \operatorname{Val}(\varphi, \mathfrak{A}, i, k)=$ 1 ; otherwise $\operatorname{Val}(H \varphi, \mathfrak{A}, i, j)=0$.

Finally, a wff $\varphi$ is said to be $\operatorname{VMTS-valid~if~and~only~if~} \operatorname{Val}(\varphi, \mathfrak{A}, i, j)=1$ for any VMTS-model $\mathfrak{A}$, possible world $i$ and time-point $j$ in $\mathfrak{A}$; and a set of wffs $\Gamma$ is VMTS-satisfiable if and only if there are a VMTS-model $\mathfrak{B}$, possible world $i$ and time-point $j$ in $\mathfrak{B}$ such that for every $\varphi \in \Gamma, \operatorname{Val}(\varphi, \mathfrak{B}, i, j)=1$. 
As already pointed out above, the set $\mathcal{D}$ of a VMTS model $\mathfrak{A}$ represents the set of concrete individuals that exist at a possible world or other. Since by the definition of an assignment in a VTMS-frame, individual variables take their values from $\mathcal{D}, \mathcal{D}$ also represents the set of possible individuals. This conception of $\mathcal{D}$ is in agreement with the interpretation of necessity as natural necessity. According to this latter sense of necessity, the only possible individuals are those having concrete existence in a naturally possible world, because they are the only ones that would instantiate a natural property. Natural properties do not count as possible individuals, because they are not abstract individuals but rather causal structures that are realized in some possible world. Not all of these structures will have to be causally realized in the actual world.

Regarding clause 6 , the following remark is in order. We have pointed out above that sortal concepts are cognitive capacities providing identity criteria only for things that exist. Thus, when a sortal concept $S$ is exercised at a possible world $w$ and time $t$, the only objects that would be identified by $S$ at $w$ and $t$ are those that exist at that word and time-point satisfying the criteria provided by $S$. Accordingly, universal reference of a relative sortal quantifier $\forall x S$, at a possible world $w$ and time $t$, will be to the set of objects existing at $w$ and $t$ that fall under the sortal concept the term " $S$ " stands for. This is what clause 6 intends to convey.

Clause 5 expresses the variable-domain interpretation of the second-order quantifiers over sortal concepts. At a possible world $w$ and time-point $t$, their domain of quantification is the set of concepts subsisting at $w$ and $t$. This set of concepts is represented in a VTMS-model by the set $\mathcal{S}(w, t)$.

By the definition of an assignment in VMTS model and clauses 5 and 6 , note that the formal system we shall characterize in the next section will constitute a free-logic regarding individual and sortal terms.

Clause 7 corresponds to our intuitive interpretation of the modal operator. A VMTS-frame will formally represent a group of worlds sharing the same causal matrix as the actual world. Thus, the most appropiate modal system of propositional logic for the operator in question is the modal logical system S5. For this reason, we have adopted such a system as part of the logic for sortals constructed in this paper.

As the reader can verify, the present semantic system allows that an object (in the sense of a value of a free individual variable) may not be identifiable by any sortal concept at all. That is, $\square \neg \exists S\left(x={ }_{S} x\right)$ is consistent in the semantic system. In other words, the semantics does not assume that every entity of the domain of discourse should fall under a sortal concept, at some possible world. This is to allow for the possibility of objects for which identity conditions cannot in principle be provided.

Another important point in the semantic system is the preservation of Leibniz's law under relative (sortal) identity. ${ }^{8}$ But the semantic validation of such a law does not mean that the semantics does not allow for contingent identities, since an approach to proper names as sortals is here presupposed. According to this approach, proper names are sortal terms standing for (sortal) concepts providing criteria for uniquely identifying or distinguishing (at most) one thing 
It is also important to note that absolute identity (viz., the concept of $x$ being the same $y$ simpliciter) can be explained in terms of the primitive logical symbols of the this paper, if we assume that every possible object falls, in principle, under some sortal concept. That is, if we assume that identity criteria can in principle be provided for every object, absolute identity could be definitionally introduced as follows: $(x=y)=_{d f} . \diamond(\exists S)\left(x={ }_{S} y\right)$, where the symbol $\diamond$ represents natural possibility (see definition 0 in the next section, for a definition of natural possibility in terms of negation and natural necessity). In other words, $x$ is the same as $y$ if and only if it is naturally possible that a sortal concept $S$ can be constructed such that $x$ is the same $S$ as $y$. It there were objects for which identity criteria cannot in principle be provided, then the definition in question cannot be philosophically sustained. This latter possibility is not precluded by conceptualism and, as pointed out above, is being taken into account in the semantics of this paper. In this case, absolute identity would have to be assumed as a primitive concept.

\section{The Formal System VMTS.}

We now proceed to characterize a formal logical system for sortals. We will show that this system is sound and complete with respect to VMTS-validity. Before formulating the system, we need to introduce the following convention and definitions:

Definition 0:

1. $\nabla \varphi=\neg \square \neg \varphi$

2. $P \varphi=\neg H \neg \varphi$

3. $F \varphi=\neg G \neg \varphi$

4. $[t] \varphi=G \varphi \& H \varphi \& \varphi$

Clearly, the operators $\diamond, P$ and $F$ here defined, correspond to the propositional operator of natural possibility, the future and the past tense propositional operator, respectively.

In the next definition, we assume that two sortal concepts are the same if and only they are co-extensive at any possible world and time-point.

Definition 1: If $L$ and $M$ are sortal term variables, $y$ and $x$ individual variables, then

$(L=M)_{y, x}=[t] \square\left(\forall y M \exists x L\left(x={ }_{L} y\right) \& \forall y L \exists x M\left(x={ }_{M} y\right)\right)$

(Note: for convenience we shall generally drop hereafter the use of the individual variables in the expression " $(L=M)_{y, x}$ " and write instead $(L=M)$, unless the context requires explicit reference to such variables). 
Convention 0: Let $\varphi$ be a wff (a)We shall use $\square \varphi$ to represent one of the wffs $G \varphi, H \varphi$ and $\square \varphi$.(b)By $\square^{+} \varphi$ we shall mean the wff $G \varphi$ if $\square \varphi$ is $H \varphi$, the wfff $H \varphi$ if $\square \varphi$ is $G \varphi$ and the wff $\square \varphi$ if $\square \varphi$ is $\square \varphi$.

Convention 1: (a) By $\odot \varphi$ we shall mean $\neg \square \neg \varphi$. So $\odot \varphi$ might represent one of the wffs $F \varphi, P \varphi$ and $\diamond \varphi$, depending clearly on what $\sqcup \varphi$ is.

We are now ready to introduce VMTS.

\section{Axioms of VMTS:}

A0. All tautologies

A1. $\forall L \exists M(L=M)$

A2. $\forall x L \exists y L\left(y={ }_{L} x\right)$

A3. $\varphi \rightarrow \forall y L \varphi$, provided $y$ does not occur free in $\varphi$

A4 $\varphi \rightarrow \forall L \varphi$, provided $L$ does not occur free in $\varphi$

A5. $x={ }_{L} x \rightarrow \exists y L\left(y={ }_{L} x\right)$, where $y$ is a variable other than $x$

A6. $\exists L(L=M) \rightarrow\left(\forall L \varphi \rightarrow \varphi^{M} / L\right)$, provided $M$ is free for $L$ in $\varphi$

A7. $x={ }_{L} y \rightarrow x={ }_{L} x$

A8. $\forall x L(\varphi \rightarrow \gamma) \rightarrow(\forall x L \varphi \rightarrow \forall x L \gamma)$

A9. $\forall L(\varphi \rightarrow \gamma) \rightarrow(\forall L \varphi \rightarrow \forall L \gamma)$

A10. $\left(\diamond\left(y={ }_{L} z\right) \vee F \diamond\left(y={ }_{R} z\right) \vee P \diamond\left(y={ }_{A} z\right)\right) \rightarrow[t] \square\left(\exists x M\left(y={ }_{M} x\right) \rightarrow\right.$ $\left.\left(y={ }_{M} z\right)\right)$, where $y$ is a variable other than $x$

A11. $x={ }_{L} y \rightarrow\left(\varphi \rightarrow \varphi^{*}\right)$, where $\varphi^{*}$ is obtained from $\varphi$ by replacing one or more free occurrences of $x$ by free occurrences of $y$.

A12. $\square \varphi \rightarrow \varphi$

A13. $\nabla \varphi \rightarrow \square \nabla \varphi$

A14. $\square(\varphi \rightarrow \sigma) \rightarrow(\square \varphi \rightarrow \square \sigma)$.

A15. $\varphi \rightarrow G P \varphi$

A16. $\varphi \rightarrow H F \varphi$

A17. $P \varphi \rightarrow H(F \varphi \vee \varphi \vee P \varphi)$

A18. $F \varphi \rightarrow G(\varphi \vee P \varphi \vee F \varphi)$

A19. $G \varphi \rightarrow G G \varphi$

A20. $H \varphi \rightarrow H H \varphi$

A21 $G(\varphi \rightarrow \sigma) \rightarrow(G \varphi \rightarrow G \sigma)$

A22 $H(\varphi \rightarrow \sigma) \rightarrow(H \varphi \rightarrow H \sigma)$

A23 $F \square \varphi \rightarrow \square F \varphi$

A24 $P \square \varphi \rightarrow \square P \varphi$

(Note: Hereafter, we shall also refer to axioms A11, A14, A21, A22, A23 and 24 as axioms $(\mathrm{LL}),(D I S T \square),(\mathrm{DIST} G),(\mathrm{DIST} H))$, and bridge axioms, respectively.

\section{Rules of VMTS:}


$\sqcup$ GenI : from $\sigma \rightarrow \triangleright_{1}\left(\gamma_{1} \rightarrow \ldots \rightarrow \square_{n}\left(\gamma_{n} \rightarrow \square \varphi\right) \ldots\right)$ infer $\sigma \rightarrow \sqcup_{1}\left(\gamma_{1} \rightarrow\right.$ $\left.\ldots \rightarrow \square_{n}\left(\gamma_{n} \rightarrow \square \forall x S \varphi\right) \ldots\right)$, provided $x$ does not occur free in $\sigma \rightarrow \square_{1}\left(\gamma_{1} \rightarrow\right.$ $\left.\ldots \rightarrow \sqcup_{n}\left(\gamma_{n} \rightarrow \square \forall x S \varphi\right) \ldots\right) ; \gamma_{1} \ldots \gamma_{n}$ are wffs, and for $0<i \leq n, \sqcup_{i} \in\{G, H$ and $\square\}$

$\square$ GenS : from $\sigma \rightarrow \square_{1}\left(\gamma_{1} \rightarrow \ldots \rightarrow \square_{n}\left(\gamma_{n} \rightarrow \square \varphi\right) \ldots\right)$ infer $\sigma \rightarrow \square_{1}\left(\gamma_{1} \rightarrow\right.$ $\left.\ldots \rightarrow \square_{n}\left(\gamma_{n} \rightarrow \square \forall S \varphi\right) \ldots\right)$, provided $S$ does not occur free in $\sigma \rightarrow \square_{1}\left(\gamma_{1} \rightarrow \ldots \rightarrow\right.$ $\left.\sqcup_{n}\left(\gamma_{n} \rightarrow \boxminus \forall S \varphi\right) \ldots\right) ; \gamma_{1} \ldots \gamma_{n}$ are wffs, and for $0<i \leq n, \sqcup_{i} \in\{G, H$ and $\square\}$

$U G(s)$ : from $\varphi$ infer $\forall L \varphi$

$U G$ : from $\varphi$ infer $\forall x L \varphi$

$M P$ : from $\varphi$ and $\varphi \rightarrow \sigma$ infer $\sigma$

$R G$ : from $\varphi$ infer $G \varphi$

$R H$ : from $\varphi$ infer $H \varphi$.

$R N$ : from $\varphi$ infer $\square \varphi$

Irr: from $(\square(\pi x \& H \neg \pi x) \rightarrow \varphi)$, infer $\varphi$, provided $\pi$ does not occur in $\varphi$

(Note: In the case of $\square$ Gen, when $n=0$, it becomes "from $\sigma \rightarrow \boxminus \varphi$ infer $\sigma \rightarrow \triangleright \forall u \varphi) "$

We shall say that a wff $\varphi$ is a theorem of VMTS (in symbols, $\vdash \varphi$ ) if and only if there are wffs $\gamma_{1} \ldots \gamma_{n}$ such that for every $i(1 \leq i \leq n), \gamma_{i}$ is either an axiom or follows from previous wffs in the sequence by one of the rules of VMTS, and $\gamma_{n}$ is $\varphi$. A wff $\varphi$ is an VMTS-theorem of $\Gamma$ (in symbols, $\Gamma \vdash \varphi$ ) if and only if there are wffs $\psi_{1} \ldots \psi_{n} \in \Gamma$ such that $\left(\psi_{1} \& \ldots \& \psi_{n}\right) \rightarrow \varphi$ is a theorem of VMTS.

Convention 2: From now on, a proof requiring reasoning in accordance with classical propositional logic will be denoted by PL.

We now state several theorems and briefly indicate how to prove them.

\section{Theorems}

T0. $\exists x L\left(x={ }_{L} y\right) \rightarrow\left(\forall x L \varphi \rightarrow \varphi^{y} / x\right)$, provided $y$ is a variable other than $x$ free for $x$ in $\varphi$

(LL, PL , UG, A3, A8)

T1. $\forall y L \varphi \leftrightarrow \forall z L \varphi^{z} / y$, provided $z$ is free for $y$ in $\varphi$ and does not occur free in $\varphi$

(T0, UG, A8, A2, A3)

T2. $\forall L \varphi \leftrightarrow \forall M \varphi^{M} / L$, provided $M$ is free for $L$ in $\varphi$ and does not occur free in $\varphi$

$(\mathrm{A} 6, U G(s), \mathrm{A} 9, \mathrm{~A} 4)$

T3. $x={ }_{L} y \rightarrow y={ }_{L} x$

$(\mathrm{LL}, \mathrm{A} 7, \mathrm{PL})$

T4. $x={ }_{L} y \rightarrow \exists z L\left(z={ }_{L} x\right)$

(A7, A5 and PL)

T5. $\exists y S\left(x={ }_{S} y\right) \rightarrow x={ }_{S} x$

Australasian Journal of Logic (12:1) 2014, Article no. 5 
(A7, PL, UG, A8, A3, definition)

T6. $\square \varphi \rightarrow \square(\varphi \& \varphi)$

(PL, R $\square$, Dist- $\square$ )

$\mathrm{T} 7 . \square(\varphi \& \psi) \rightarrow \square(\psi \& \varphi)$

$(P L, \mathrm{R} \square$, Dist $-\square)$

T8. $(\square(\varphi \& \psi) \& \square(\psi \& \delta)) \rightarrow \square(\varphi \& \delta)$

(PL, R $\square$, Dist- $\square$ )

T9. $\diamond\left(x={ }_{S} y\right) \rightarrow \diamond\left(y={ }_{S} x\right)$

(T3, R $\square$, Dist $\square, P L$, de finition)

T10. $F \diamond\left(x={ }_{S} y\right) \rightarrow F \diamond\left(y={ }_{S} x\right)$

(T9, PL, RG, DistG, PL , definition)

T11. $P \diamond\left(x={ }_{S} y\right) \rightarrow P \diamond\left(y={ }_{S} x\right)$

(T9, PL, RH,Dist H,PL, definition)

T12. $(G \square \varphi \rightarrow G \square(\varphi \& \varphi)$

(T6, PL, RG, Dist $G$ )

T13. $(H \square \varphi \rightarrow H \square(\varphi \& \varphi)$

(T6, PL, RH, Dist $H)$

T14. $(\square \varphi \& \diamond \gamma) \rightarrow \diamond(\varphi \& \gamma)$

(PL, R $\square$, Dist- $\square, P L)$

$\mathrm{T} 15(H \varphi \& P \gamma) \rightarrow P(\varphi \& \gamma)$

(PL, RG, Dist $G, P L)$

T16. $(G \varphi \& F \gamma) \rightarrow F(\varphi \& \gamma)$

$(\mathrm{PL}, \mathrm{RH}, \operatorname{Dist} H, P L)$

T17. $\left(\diamond\left(x==_{M} y\right) \& \diamond\left(\begin{array}{ll}y={ }_{L} & z\end{array}\right)\right) \rightarrow \diamond\left(\begin{array}{ll}x={ }_{L} & z\end{array}\right)$

(A10, T9, T4, T6, T14, PL, R $\square, \mathrm{LL})$

$\mathrm{T} 18 . F(\square \varphi \& \diamond \gamma) \rightarrow F \diamond(\varphi \& \gamma)$

$(\mathrm{T} 14, \mathrm{R} G, \mathrm{Dist} G,, P L)$

$\mathrm{T} 19 . P(\square \varphi \& \diamond \gamma) \rightarrow P \diamond(\varphi \& \gamma)$

(T14, RH,Dist $H, P L)$

$\mathrm{T} 20 . \diamond\left(x={ }_{S} y\right) \& F \diamond\left(y={ }_{L} z\right) \rightarrow F \diamond\left(x={ }_{L} z\right)$

(A10, T9, T4, T6, T16, PL, R $\square, \mathrm{RG}, \mathrm{LL})$

$\mathrm{T} 21 . \diamond\left(x={ }_{S} y\right) \& P \diamond\left(y={ }_{L} z\right) \rightarrow P \diamond\left(x={ }_{L} z\right)$

(A10, T9, T4, T6, T15, PL, R $\square, \mathrm{RG}, \mathrm{LL})$

$\mathrm{T} 22 . F \diamond\left(x={ }_{S} y\right) \& P \diamond\left(y={ }_{L} z\right) \rightarrow P \diamond \exists S\left(x={ }_{L} z\right)$

(A10, T9, T4, T15, PL, R $\square, \mathrm{RG}, \mathrm{LL})$

$\mathrm{T} 23 . P \diamond\left(x={ }_{S} y\right) \& F \diamond \exists S\left(y={ }_{L} z\right) \rightarrow F \diamond\left(x={ }_{S} z\right)$

( A10, T9, T4, T16, PL, R $\square, \mathrm{RG}, \mathrm{LL}$ )

$\mathrm{T} 24 . P \diamond\left(x={ }_{S} y\right) \& P \diamond\left(y={ }_{L} z\right) \rightarrow P \diamond\left(x={ }_{L} z\right)$

(A10, T9, T4,T6, T17, PL, R $\square, \mathrm{RH}, \mathrm{LL})$

$\mathrm{T} 25 . F \diamond\left(x={ }_{S} y\right) \& F \diamond\left(y={ }_{L} z\right) \rightarrow F \diamond \exists S\left(x={ }_{L} z\right)$

(A10, T9, T4, T16, PL, R $\square$, RG, LL )

T26. $\square \varphi \rightarrow \square \square \varphi$

(A12, A13, PL, and definitions)

T27. $P \diamond\left(x={ }_{S} y\right) \& \diamond\left(y={ }_{L} z\right) \rightarrow P \diamond \exists S\left(x={ }_{L} z\right)$

( A10, T9, T4, T6, T14, PL, R $\square, \mathrm{RH}$ LL )

T28. $F \diamond\left(x={ }_{S} y\right) \& \diamond\left(y={ }_{L} z\right) \rightarrow F \diamond\left(x=_{L} z\right)$ 
(A10, T9, T4, T6, T14, PL, R $\square, \mathrm{RG}, \mathrm{LL}$ )

T29. $([t] \square(\varphi \& \psi) \&[t] \square(\psi \& \delta)) \rightarrow[t] \square(\varphi \& \delta)$

(T8, RG, RH, Dist $G$, Dist $H, \mathrm{PL})$

T30. $([t] \square(\varphi \& \psi)) \rightarrow[t] \square(\psi \& \varphi)$

(T7, R $G, \mathrm{R} H, \operatorname{Dist} G$, Dist $H, \mathrm{PL})$

T31. $(\square \varphi \& \square \psi) \rightarrow \square(\varphi \& \psi)$

T32. $\square G \varphi \rightarrow G \square \varphi$

(PL, A15, A24, T16, A16, T14)

T33. $G \square \varphi \rightarrow \square G \varphi$

(PL, A23, A13, RG, A12, RN)

T34. $\square H \varphi \rightarrow H \square \varphi$

(PL, A16, A23, T15, A15, T14)

T35. $H \square \varphi \rightarrow \square H \varphi$

(PL, A24, A13, RH, A12, RN)

Derived rule 1 ( Replacement rule) : if $\vdash \varphi \leftrightarrow \psi$, then $\vdash \delta \leftrightarrow \delta^{*}$, where $\delta^{*}$ is the result of replacing one or more occurrences of $\varphi$ by $\psi$ in $\delta$.

Proof: by strong induction on the complexity of $\delta$

Derived rule 2 (a) if $\vdash \sigma \rightarrow \square_{1}\left(\varphi_{1} \rightarrow \ldots \square_{n}\left(\varphi_{n} \rightarrow \square_{n+1}\left(\exists x S\left(x=_{S} y\right) \rightarrow \psi\right) \ldots\right)\right.$, then $\vdash \sigma \rightarrow \triangleright_{1}\left(\varphi_{1} \rightarrow \ldots \sqcup_{n}\left(\varphi_{n} \rightarrow \square_{n+1} \forall y S \psi\right) \ldots\right)$, provided $y$ is an individual variable that does not occur free in $\sigma \rightarrow \square_{1}\left(\varphi_{1} \rightarrow \ldots \square_{n}\left(\varphi_{n} \rightarrow \square_{n+1} \forall y S \psi\right) \ldots\right)$ and for every $i \in \omega, \square_{i+1} \in\{G, H, \square\}$.

Proof: by $\square$ Gen $I$, derived rule 1 and the fact that (by A2, A8, UG and PL) $\vdash \forall y S\left(\exists x S\left(x={ }_{S} y\right) \rightarrow \psi\right) \longleftrightarrow \forall y S \psi$.

Derived rule 2(b) if $\vdash \sigma \rightarrow \square_{1}\left(\varphi_{1} \rightarrow \ldots . \square_{n}\left(\varphi_{n} \rightarrow \square_{n+1}(\exists L(L=M) \rightarrow \psi) \ldots\right)\right.$, then $\vdash \sigma \rightarrow \triangleright_{1}\left(\varphi_{1} \rightarrow \ldots \square_{n}\left(\varphi_{n} \rightarrow \square_{n+1} \forall M \psi\right) \ldots\right)$, provided $M$ is a sortal term variable that does not occur free in $\sigma \rightarrow \square_{1}\left(\varphi_{1} \rightarrow \ldots \square_{n}\left(\varphi_{n} \rightarrow \square_{n+1} \forall M \psi\right) \ldots\right)$ and for every $i \in \omega, \triangleright_{i+1} \in\{G, H, \square\}$.

Proof: similar to the above proof.

Derived rule 3: If $\vdash \sigma \rightarrow \square_{0}\left(\varphi_{1} \rightarrow \ldots \square_{n-1}\left(\varphi_{n} \rightarrow \square_{n} \neg \psi\right) \ldots\right)$, then $\vdash \psi \rightarrow$ $\square_{n}^{+}\left(\varphi_{n} \rightarrow \ldots . \square_{1}^{+}\left(\varphi_{1} \rightarrow \square_{0}^{+} \neg \sigma\right) \ldots\right)$. for every $i \in \omega, 0<i \leq n, \square_{i} \in\{G, H$ and $\square$.

Proof: Assume hypothesis. By A12-13, A15, A16, RN, RG, RH, Dist $G$, Dist $H$ and Dist $\square$, it can be shown by weak induction that $\vdash \odot_{j-1}\left(\varphi_{j} \& \odot_{j}\left(\varphi_{j+1}\right.\right.$ $\left.\& \ldots \& \odot_{n-1}\left(\varphi_{n} \& \odot_{n} \psi\right) \ldots\right) \rightarrow\left(\varphi_{j-1} \rightarrow \sqcup_{j-2}^{+}\left(\varphi_{j-2} \rightarrow \ldots . \odot_{1}^{+}\left(\varphi_{1} \rightarrow \odot_{0}^{+} \neg \sigma\right) \ldots\right)\right.$. Then by $j=n+1, \vdash \odot_{n} \psi \rightarrow\left(\varphi_{n} \rightarrow \triangleright_{n-1}^{+}\left(\varphi_{n-1} \rightarrow \ldots \sqcup_{1}^{+}\left(\varphi_{1} \rightarrow \triangleright_{0}^{+} \neg \sigma\right) \ldots\right)\right.$. By RN, RG or RH ; Dist $G$, Dist $H$ or Dist $\square, \vdash \square_{n}^{+} \odot_{n} \psi \rightarrow \sqcup_{n}^{+}\left(\varphi_{n} \rightarrow \sqcup_{n-1}^{+}\left(\varphi_{n-1} \rightarrow\right.\right.$ $\left.\ldots . \triangleright_{1}^{+}\left(\varphi_{1} \rightarrow \square_{0}^{+} \neg \sigma\right) \ldots\right)$ and so by A12-13, and A15-A16, $\vdash \psi \rightarrow \square_{n}^{+}\left(\varphi_{n} \rightarrow\right.$ $\square_{n-1}^{+}\left(\varphi_{n-1} \rightarrow \ldots . \sqcup_{1}^{+}\left(\varphi_{1} \rightarrow \sqcup_{0}^{+} \neg \sigma\right) \ldots\right)$

As the reader might have noticed, the principle $\forall L \varphi \rightarrow \forall x M \varphi^{M} / L$ (where $M$ is free for $L$ in $\varphi$ ), which relates second-order quantification with first-order 
relative quantification, is not among the above theorems. As the reader can verify, such a formula is not valid in the semantics, and a counter-example can be found in the formula $\left(\forall F \exists K \exists y K\left(x={ }_{F} y\right) \rightarrow \forall x M \exists K \exists y K\left(x={ }_{M} y\right)\right.$. By the soundness result in the following section, it follows that the principle in question cannot be a theorem of VMTS either.

Another important aspect of the above formal system concerns the interaction of the alethic modality with the two kinds of quantifiers. Both the Barcan formula and its converse are neither valid in the semantics of the present paper nor theorems of the formal system, given the soundness proof in the following section. That is, formulas of the form $\forall L \square \varphi \rightarrow \square \forall L \varphi, \square \forall L \varphi \rightarrow \forall L \square \varphi$, $\forall x L \square \varphi \rightarrow \square \forall x L \varphi$ and $\square \forall x L \varphi \rightarrow \forall x L \square \varphi$ are neither VMTS-valid nor VMTS theorems. In the case of the second-order quantification over sortal concepts, the reason is that the value of the function $\mathcal{S}$ of a VMTS-model might change from one possible world and time-point to another possible world and/or timepoint. This possible variation does not guarantee that the set of sortal concepts at a certain world and time-point would be a subset of the set of concepts of another world and time-point, and vice-versa. This condition renders both the Barcan formula and its converse VMTS-invalid for second-order quantifiers.

In the case of (first-order) relative sortal quantification, notice that the extension of a sortal concept might vary from one possible world to another, and at a possible world, from one time-interval to another. This variation leaves open the possibility that the extension of the sortal concept at a possible world and time point might not be a subset of the extension of that concept at another possible world and/or time point, and vice-versa. Since (first-order) relative sortal quantification depends on the extension of the sortal concept on which such a quantification is based, the VMTS-validity of the corresponding Barcan formula and its converse is not guaranteed.

Now, the following theorems can be proved in the formal system VMTS :

- $\square \exists L(L=M) \rightarrow(\forall L \square \varphi \rightarrow \square \forall L \varphi) \quad$ (by A6, RN,Dist $\square$, A12, A13, UG(s), A9, A4, T26 and PL)

- $\square \exists L(L=M) \rightarrow(\square \forall L \varphi \rightarrow \forall L \square \varphi)$ (by A6, RN, Dist $\square, \mathrm{UG}(\mathrm{s})$, A9, A4 and PL).

These theorems establish sufficient conditions for deriving the Barcan formula and its converse. (Similar theorems for (first-order) relative sortal quantifiers can be derived in VMTS.) Thus, in the case of the second-order quantifiers, the Barcan formula and its converse can be proved under the assumption that, by natural necessity, every sortal term stands for a sortal concept. In other words, if the laws of the formation of concepts as determined by the natural properties implies that a stage of concept formation can be reached at which every sortal predicate would stand for a concept, then the Barcan formula and its converse would hold.

The possibility of the above stage of concept formation has been taken into account in holistic conceptualism (see, for example, in Cocchiarella (2007)). 
Holistic conceptualism is a modern variant of conceptualism that looks at concept construction as a process of different stages in which conceptual structures at a later stage are in general not explicitly definable or reducible to those at the earlier stages they presuppose (as conceptually prior bases for their constructions). These earlier stages are retained as still important and useful parts of the overall conceptual framework. A final stage at which all possible predicates stand for a concept is postulated and justified by holistic conceptualism. Clearly, the logical system characterized in this paper is neutral with respect to this stage of concept-formation.

Australasian Journal of Logic (12:1) 2014, Article no. 5 


\section{Completeness and soundness of VMTS.}

We now show that system VMTS is sound and complete. First, we assume the

following conventions and define certain notions most of which are instrumental in the completeness proof.

Convention 3: Let $\varphi$ be a wff. By $* \varphi$ we shall mean any wff of the form $\odot_{1}\left(\gamma_{1}\right.$ $\left.\& \ldots \odot_{n-1}\left(\gamma_{n-1} \& \odot \varphi\right) \ldots.\right)$, where $\gamma_{1} \ldots \gamma_{n}$ are wffs, for $0<i \leq n, \odot_{i} \in\{P, F$ and $\diamond\}$ and $\odot \in\{P, F$ and $\diamond\}$. (Note: when $n=1$, then $* \varphi$ is $\odot \varphi$ ).

Convention 4: If $* \varphi$ is $\odot_{1}\left(\gamma_{1} \& \ldots \odot_{n}\left(\gamma_{n} \& \odot \varphi\right) \ldots.\right)$, then in any given context in which a wff $* \varphi$ occurs, then $* \psi$ in the same context will be $\odot_{1}\left(\gamma_{1} \& \ldots \odot_{n}\left(\gamma_{n}\right.\right.$ $\& \odot \psi) \ldots$.$) unless otherwise indicated.$

\section{Definition 2:}

Let $\Gamma$ be a set of wffs.

1. $\Gamma$ is VMTS-consistent if and only if there are not wffs $\psi_{1} \ldots \psi_{n} \in \Gamma$ such that $\left(\psi_{1} \& \ldots \& \psi_{n}\right) \rightarrow(\varphi \& \neg \varphi)$ is a theorem of VMTS.

2. $\Gamma$ is $\omega$-complete if and only if $\Gamma$ satisfies the following three clauses:(a) if $\exists x S \varphi \in \Gamma$, then there is a variable $y$ other than $x$ which is free for $x$ in $\varphi$ such that $\left(\exists x S\left(x=_{S} y\right) \& \varphi^{y} / x\right) \in \Gamma$; (b) for all wff $\varphi$, if $\exists S \varphi \in \Gamma$, then there is a sortal term $T$ other than $S$ which is free for $S$ in $\varphi$ such that $\left(\exists S(S=T) \& \varphi^{T} / S\right) \in \Gamma ; \quad(c)$ for all wff $\varphi$, if $* \exists x S \varphi \in \Gamma$, then there is a variable $y$ other than $x$ which is free for $x$ in $\varphi$ such that $*\left(\exists x S\left(x={ }_{S} y\right)\right.$ $\left.\& \varphi^{y} / x\right) \in \Gamma$; and $(d)$ for all wff $\varphi$, if $* \exists S \varphi \in \Gamma$, then there is a variable $T$ other than $S$ which is free for $S$ in $\varphi$ such that $*\left(\exists S(S=T) \& \varphi^{T} / S\right) \in \Gamma$

3. $\Gamma$ is irreflexive if and only if both (a) for all wff $\varphi$, if $* \varphi \in \Gamma$, then there is an one-place predicate variable $R$ which does not occur in $* \varphi$ and an individual variable $x$ such that $*(\square(R x \& H \neg R x) \& \varphi) \in \Gamma$; and (b) there is an one-place predicate variable $R$ and individual variable $x$ such that $\square(R x \& H \neg R x) \in \Gamma$

4. $\Gamma$ is a VMTS-maximally consistent set of wffs if and only if $\Gamma$ is VMTSconsistent and for every wff $\varphi$, either $\varphi \in \Gamma \operatorname{or} \neg \varphi \in \Gamma$.

5. $\Gamma$ is a maxc set of wffs if and only if $\Gamma$ is an irreflexive, maximally consistent $\omega$-complete set of wffs.

Definition 3: Let $\varphi$ be a wff , $\alpha$ an individual or sortal term variable. By recursion, we shall define the expression " $\left[\varphi^{(\alpha)}\right]$ ", which intuitively should be understood as the result of rewriting all bound occurrences of $\alpha$ by variables new to $\varphi$ of the same type as $\alpha$.

- If $\varphi$ is an atomic wff, then $\left[\varphi^{(\alpha)}\right]=\varphi$ 
- If $\varphi$ is of the form $\neg \psi$, then $\left[\varphi^{(\alpha)}\right]=\neg\left[\psi^{(\alpha)}\right]$

- If $\varphi$ is of the form $\psi \rightarrow \gamma$, then $\left[\varphi^{(\alpha)}\right]=\left[\psi^{(\alpha)}\right] \rightarrow\left[\gamma^{(\alpha)}\right]$

- If $\varphi$ is of the form $\forall z S \psi$, then

$$
\left[\varphi^{(\alpha)}\right]=\left\{\begin{array}{l}
\forall z S\left[\psi^{(\alpha)}\right], \text { if } z \text { is not } \alpha \\
\forall k S\left[\psi^{(\alpha)}\right]^{k} / \alpha, \text { if } z \text { is } \alpha \\
\text { where } k \text { is the first individual variable new to both }\left[\psi^{(\alpha)}\right] \text { and } \varphi .
\end{array}\right.
$$

- If $\varphi$ is of the form $\forall S \psi$, then

$$
\left[\varphi^{(\alpha)}\right]=\left\{\begin{array}{l}
\forall S\left[\psi^{(\alpha)}\right], \text { if } S \text { is not } \alpha \\
\forall H\left[\psi^{(\alpha)}\right]^{H} / \alpha, \text { if } S \text { is } \alpha \\
\text { where } H \text { is the first sortal term variable new to both }\left[\psi^{(\alpha)}\right] \text { and } \varphi .
\end{array}\right.
$$

- If $\varphi$ is of the form $\square \psi$, then $\left[\varphi^{(\alpha)}\right]=\square\left[\psi^{(\alpha)}\right]$

Definition 4: For every maxc $\Gamma, \Sigma, \Gamma R_{\square} \Sigma$ if and only if for every wff $\varphi$, if $\sqcup \varphi \in \Gamma$, then $\varphi \in \Sigma \quad($ where $\square \in\{G, H, \square\})$

We shall now state several lemmas instrumental for the completeness or soundness proofs in this section. We omit the proofs for lemmas 0-9 because they are similar to those for the same or analogous lemmas in Freund ((2007) pp. 585-87).

Lemma 0: For any maxc $\Gamma$ and $\Sigma$, and for any wff $\varphi$

I.The following are equivalents

(a) whenever $\varphi \in \Gamma$, we have $P \varphi \in \Sigma$

(b) whenever $\varphi \in \Sigma$, we have $F \varphi \in \Gamma$

(c) whenever $G \varphi \in \Gamma$, we have $\varphi \in \Sigma$

(d) whenever $H \varphi \in \Sigma$, we have $\varphi \in \Gamma$

II.The following are equivalents:

(e)whenever $\square \varphi \in \Sigma$, we have $\varphi \in \Gamma$

$(f)$ whenever $\varphi \in \Gamma$, we have $\nabla \varphi \in \Sigma$

Lemma $1 . R_{\square}$ is an equivalence relation in the set of maxc sets.

Definition 5: For every maxc set $\Gamma,[\Gamma]_{R_{\square}}$ is the equivalence class of $\Gamma$ determined by $R_{\square}$ in the set of maxc sets.

Definition 6 :For every maxc $\Gamma, \Sigma, \Gamma \simeq_{G} \Sigma$ if and only if $\Gamma=\Sigma$ or $\Gamma R_{G} \Sigma$ or $\Sigma R_{G} \Gamma$

Lemma 2. (a) $R_{G}$ is transitive (b) the restriction of $R_{G}$ to an arbitrary set of maxc sets of wffs is an irreflexive relation (c) $R_{G}$ is left- and right-serial (d) $\simeq_{G}$ is an equivalence relation in the set of maxc sets.

Australasian Journal of Logic (12:1) 2014, Article no. 5 
Definition: Let $[\Gamma]_{\simeq_{G}}$ the equivalence class of $\Gamma$ determined by $\simeq_{G}$ in the set of maxc consistent sets.

Lemma 3 . The relation $R_{G}$ is a serial order on every equivalence class $[\Gamma]_{\simeq_{G}}$

Lemma 4. If $\Gamma$ and $\Sigma$ are maxc sets of wffs such that $\Gamma \simeq_{G} \Sigma$ and there is a one-place predicate variable $P$ and an individual variable $x$ such that

$(P x \& H \neg P x) \in \Gamma \cap \Sigma$, then $\Gamma=\Sigma$.

Lemma 5. For any individual variable $x, \vdash\left[\gamma^{(x)}\right] \leftrightarrow \gamma$

Lemma 6. For any sortal term variable $S, \vdash\left[\gamma^{(S)}\right] \leftrightarrow \gamma$.

Note that: (i) If $x$ is free for $y$ in $\gamma$, then $\left[\gamma^{x} / y^{(x)}\right]$ is $\left[\gamma^{(x)}\right]^{x} / y$ and so by Lemma 5 ,

$\vdash\left[\gamma^{(x)}\right]^{x} / y \leftrightarrow \gamma^{x} / y$; and (ii) If $S$ is free for $H$ in $\gamma$, then $\left[\gamma^{S} / H^{(S)}\right]$ is $\left[\gamma^{(S)}\right]^{S} / H$ and so by Lemma $6, \vdash\left[\gamma^{(S)}\right]^{S} / H \leftrightarrow \gamma^{S} / H$

Lemma 7. For any wff $\varphi$, If $\mathfrak{A}=<<\mathcal{D}, \mathcal{S}, \mathcal{W}, \mathcal{T}, \mathcal{R}>, \mathcal{A}>$, is a VMTS-model and $y$ is an individual variable free for $x$ in $\varphi$, then for every $j \in \mathcal{W}, t \in \mathcal{T}$, $\operatorname{Val}(\varphi, \mathfrak{A}(\mathcal{A}(y) / x), j, t)=1$ if and only if $\operatorname{Val}\left(\varphi^{y} / x, \mathfrak{A}, j, t\right)=1$.

Lemma 8. For any wff $\varphi$, If $\mathfrak{A}=<<\mathcal{D}, \mathcal{S}, \mathcal{W}, \mathcal{T}, \mathcal{R}>, \mathcal{A}>$ is a VMTS-model and $M$ is a sortal term variable free for $L$ in $\varphi$, then for every $j \in \mathcal{W}, t \in \mathcal{T}$, $\operatorname{Val}(\varphi, \mathfrak{A}(\mathcal{A}(L) / M), j, t)=1$ if and only if $\operatorname{Val}\left(\varphi^{M} / L, \mathfrak{A}, j, t\right)=1$.

Lemma 9: If $\varphi$ is a wff, $\mathfrak{A}=<<\mathcal{D}, \mathcal{S}, \mathcal{W}, \mathcal{T}, \mathcal{R}>, \mathcal{A}>$ and $\mathfrak{B}=<<\mathcal{D}$, $\mathcal{S}, \mathcal{W}, \mathcal{T}, \mathcal{R}>, \mathcal{B}>$ are VMTS-models such that $\mathcal{A}$ and $\mathcal{B}$ agree on all variables occurring free in $\varphi$,then $\operatorname{Val}(\varphi, \mathfrak{A}, i, j)=1$ if and only if $\operatorname{Val}(\varphi, \mathfrak{B}, i, j)=1$, for every $i \in \mathcal{W}$ and $j \in \mathcal{T}$.

Metatheorem I (soundness theorem): if $\varphi$ is a theorem of VMTS, then $\varphi$ is VMTS-valid.

Proof: By induction on the length of proofs. Directly from the semantic clauses, it can be shown the validity of axioms A0, A1, A2, A5, A7-A10, A14-A16, A2124 , and rules RG, RH, RN, UG, UG(s), $\square$ GenI, $\square$ GenS and MP. The validity of A19-A20 and A17-18 follows from the transitivity and connectivity of the later-than relation, respectively, and the semantic clauses; and similarly, that of A12-13 from the assumption that the accessibility relation is reflexive and euclidean, respectively. For axioms A3 and A4, lemma 9 is needed, and for A6 and A11, lemma 8, in addition to the semantic clauses.

For the case of $\operatorname{Irr}$, assume first that $(\square(\pi x \& H \neg \pi x) \rightarrow \varphi)$ is valid and that $\varphi$ is not valid (where $\pi$ does not occur in this latter formula). Therefore, there is a VMTS-model $\mathfrak{A}=\langle<\mathcal{D}, \mathcal{S}, \mathcal{W}, \mathcal{T}, \mathcal{R}>, \mathcal{A}>$ and $w \in \mathcal{W}, t \in \mathcal{T}$, such that $\operatorname{Val}(\varphi, \mathfrak{A}, w, t)=0$.Now, let $\mathfrak{A}^{*}=\left\langle<\mathcal{D}, \mathcal{S}, \mathcal{W}, \mathcal{T}, \mathcal{R}>, \mathcal{A}^{*}>\right.$, where $\mathcal{A}^{*}$ is 
an assignment to all variables such that: $\mathcal{A}^{*}$ is like $\mathcal{A}$ except for what it assigns to the monadic predicate variable $\pi$. In the case of $\pi, \mathcal{A}^{*}$ assigns that function $f_{\pi} \in \wp(\mathcal{D})^{\mathcal{W} \times \mathcal{T}}$ such that, for every $(j, k) \in \mathcal{W} \times \mathcal{T}$,

$$
f_{\pi}(j, k)=\left\{\begin{array}{l}
A(\pi)((j, k)) \cup\{A(x)\}, \text { if either } t=k \text { or } t R k \\
A(\pi)((j, k))-\{A(x)\} . \text { otherwise }
\end{array}\right.
$$

By Lemma $\left.9, \operatorname{Val}\left(\varphi, \mathfrak{A}^{*}, w, t\right)=0\right)$, given the assumption that $\operatorname{Val}(\varphi, \mathfrak{A}, w, t)=$ $0)$. By the irreflexivity of the $\mathcal{R}$-relation, $\operatorname{Val}\left(\square(\pi x \& H \neg \pi x), \mathfrak{A}^{*}, w, t\right)=1$. So $(\square(\pi x \& H \neg \pi x) \rightarrow \varphi)$ is not valid.

Lemma 10 . If $\Gamma$ is maxc and $\odot \gamma \in \Gamma$, then there is a maxc $\Sigma$ such that $\gamma \in \Sigma$ and $\{\psi \mid \sqcup \psi \in \Gamma\} \subseteq \Sigma$, (where $\sqcup \in\{G, H$ and $\square\}$ ).

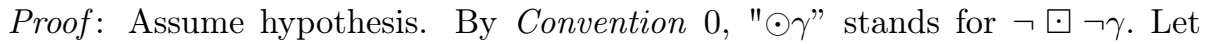
$\delta_{1} \ldots \delta_{n} \ldots$ be an ordering of wffs of the form either $\exists y S \varphi, * \varphi$ or $\exists S \varphi$. Recursively define a sequence of wffs $\psi_{0} . . \psi_{n} \ldots$ as follows.

i) $\psi_{0}=\gamma$

ii) If $\odot\left(\psi_{0} \& \ldots \& \psi_{n} \& \delta_{n+1}\right) \notin \Gamma$, then $\psi_{n+1}=\psi_{n}$

iii) If $\odot\left(\psi_{0} \& \ldots \& \psi_{n} \& \delta_{n+1}\right) \in \Gamma$, then

iiia) if $\delta_{n+1}$ is of the form $\left.\left.\exists y S \varphi, \psi_{n+1}=\left(\exists y S\left(y={ }_{S} x\right) \& \varphi^{x} / y\right)\right)\right\}$

where $x$ is the first variable other than $y$ which is free for $y$ in $\varphi$ such that $\odot\left(\psi_{0}\right.$ $\left.\& \ldots \& \psi_{n} \&\left(\exists y S\left(y={ }_{S} x\right) \& \varphi^{x} / y\right)\right) \in \Gamma$,

iiib) if $\delta_{n+1}$ is of the form $* \varphi$, then

$$
\psi_{n+1}=\left\{\begin{array}{l}
\left.*\left(\square(R x \& H \neg R x) \& \exists y S\left(y={ }_{S} z\right) \& \sigma^{z} / y\right)\right), \text { if } \varphi \text { is } \exists y S \sigma, \text { for } \\
\text { some wff } \sigma ; \\
\left.*\left(\square(R x \& H \neg R x) \& \exists S(S=T) \& \sigma^{T} / S\right)\right), \text { if } \varphi \text { is } \exists S \sigma, \text { for } \\
\text { some wff } \sigma ; \\
*(\square(R x \& H \neg R x) \& \varphi) \text { otherwise }
\end{array}\right.
$$

where (1) both $R$ is the first predicate variable and $x$ the first individual variable which do not occur in $\odot\left(\psi_{0} \& \ldots \& \psi_{n} \& \delta_{n+1}\right)$, if $\varphi$ is not of the form $\exists y S \sigma$ or $\exists S \sigma$, for some wff $\sigma$; (2) if $\varphi$ is of the form $\exists y S \sigma$, for some wff $\sigma, z$ is the first individual variable other than $y$ which is free for $y$ in $\sigma$ such that $\odot\left(\psi_{0} \&\right.$ $\left.\ldots \& \psi_{n} \& *\left(\exists y S\left(y={ }_{S} z\right) \& \sigma^{z} / y\right)\right) \in \Gamma$ and $R$ is the first predicate variable which do not occur in $\odot\left(\left(\gamma_{0} \& \ldots \& \gamma_{n}\right) \& *\left(\exists y S\left(y==_{S} z\right) \& \sigma^{z} / y\right)\right)$ and $x$ the first individual variable such that $\odot\left(\left(\gamma_{0} \& \ldots \& \gamma_{n}\right) \& *(\square(R x \& H \neg R x) \&\right.$ $\left.\left.\exists y S\left(y={ }_{S} z\right) \& \sigma^{z} / y\right)\right) \in \Gamma$; (3) if $\varphi$ is of the form $\exists S \sigma$, for some wff $\sigma, T$ is the first sortal variable other than $S$ which is free for $S$ in $\sigma$ such that $\odot\left(\psi_{0} \&\right.$ $\left.\ldots \& \psi_{n} \& *\left(\exists S(S=T) \& \sigma^{T} / S\right)\right) \in \Gamma$ and $R$ is the first predicate variable which does not occur in $\odot\left(\left(\gamma_{0} \& \ldots \quad \& \gamma_{n}\right) \& *\left(\exists S(S=T) \& \sigma^{T} / S\right)\right)$ and $x$ the first individual variable such that $\odot\left(\left(\gamma_{0} \& \ldots \& \gamma_{n}\right) \& *(\square(R x \& H \neg R x) \&\right.$ $\left.\left(\exists S(S=T) \& \sigma^{T} / S\right)\right) \in \Gamma$.

iiic) if $\delta_{n+1}$ is of the form $\exists S \varphi$, then $\psi_{n+1}=\left(\exists S(S=L) \& \varphi^{L} / S\right)$ (where $L$ is the first sortal term variable such that $\odot\left(\psi_{0} \& \ldots \& \psi_{n} \&\left(\exists S(S=L) \& \varphi^{L} / S\right)\right) \in$ $\Gamma$.

Australasian Journal of Logic (12:1) 2014, Article no. 5 
(Note: For a justification of the existential claims regarding variables in the above clauses (iiia)-(iiic), see proof of Proposition I, in the Appendix.)

Now, on the basis of the above recursion, it can be easily shown that for all $n \in \omega, \odot\left(\psi_{0} \& \ldots \& \psi_{n}\right) \in \Gamma$ and then that for all $n \in \omega,\left\{\psi_{0} \& \ldots \& \psi_{n}\right\}$ is consistent. Let $\Sigma=\{\varphi \mid \boxminus \varphi \in \Gamma\} \cup\left\{\psi_{n}: n \in \omega\right\}$. By reductio ad absurdum, we will show that $\Sigma$ is consistent.

So suppose $\Sigma$ is not consistent. Then there are $n, m \in \omega$ such that $\left\{\varphi_{0}, \ldots\right.$, $\left.\varphi_{n}, \psi_{0}, \ldots, \psi_{m}\right\} \subseteq \Sigma$ and $\vdash \neg\left(\varphi_{0} \& \ldots . \quad \& \varphi_{n} \& \psi_{0} \& \ldots \& \psi_{m}\right)$. So, by R $\bullet$ and definitions, $\vdash \neg \odot\left(\varphi_{0} \& \ldots \& \& \varphi_{n} \& \psi_{0} \& \ldots \& \psi_{n}\right)$; then by maximality of $\Gamma, \neg \odot\left(\varphi_{0} \& \ldots \& \varphi_{n} \& \psi_{0} \& \ldots \& \psi_{n}\right) \in \Gamma$. On the other hand, (given that $\left\{\square \varphi_{0} \&\right.$ $\left.\ldots . \& \square \varphi_{n}\right\} \subseteq \Gamma, \Gamma$ is maxc, and $\left.\odot\left(\psi_{0} \& \ldots \& \psi_{n}\right) \in \Gamma\right)$, by T14-16 $\odot\left(\varphi_{0} \& \ldots \&\right.$ $\left.\varphi_{n} \& \psi_{0} \& \ldots \& \psi_{n}\right) \in \Gamma$, which is impossible by the consistency of $\Gamma$. Therefore, $\Sigma$ is consistent.

We assume without loss of generality that there are one-place predicate variables not occurring in $\Sigma$. Otherwise for each $m \in \omega$, replace the $m$-th oneplace predicate variable in all the wffs in $\Sigma$ by the $2 \mathrm{~m}$-th one-place predicate variable. It can be easily shown that the replacement set for $\Sigma$ is consistent if $\Sigma$ is consistent. In the ordering of the one-place predicate variables, let $R$ be the first of the predicate variables not occurring in $\Sigma$. Let $K=\Sigma \cup\{\square(R x \& H \neg R x)\}$. By the $I r r$ rule and PL, $K$ is consistent. By Lindenbaum's method, extend $K$ to a maximally consistent set $K^{*}$. Since $\left\{\psi_{n}: n \in \omega\right\} \subseteq K^{*}, K^{*}$ is $\omega$-complete. It is clearly irreflexive as well. Also by construction, $\gamma \in K^{*}$ and $\{\varphi \mid \triangleright \varphi \in \Gamma\} \subseteq$ $K^{*}$.

Metatheorem II (Completeness Theorem for VMTS): If $\Delta$ is VMTS-consistent, then $\Delta$ is VMTS-satisfiable

Proof : Assume the hypothesis of the theorem. Without loss of generality, assume there are denumerably many individual variables $y_{1} \ldots y_{n} \ldots$,denumerably many sortal term variables $L_{0} \ldots L_{n} \ldots$ and denumerably one-place predicate variables $R_{0} \ldots R_{n} \ldots$ which do not occur in $\Delta$.(Otherwise for each $k, m, n \in \omega$, replace the $k$-th individual variable, the $m$-th-sortal term variable and $n$-th oneplace predicate variable in all the wffs in $\Delta$ by the $2 k$-th individual variable, the $2 \mathrm{~m}$-th sortal term variable and $2 \mathrm{n}$-th one-place predicate variable, respectively. It can then be easily shown that $\Delta$ is satisfiable if and only if the replacement set for $\Delta$ is and that the replacement set for $\Delta$ is consistent if $\Delta$ is consistent).

Let $R^{+}$be the first predicate variable not occurring in $\Delta$. By assumption and the $\operatorname{Irr}$ rule, $\Delta \cup\left\{\square\left(R^{+} x \& H \neg R^{+} x\right)\right\}$ is consistent. Define a chain of sets $\Gamma_{0} . . \Gamma_{n} \ldots$ as follows.

1) $\Gamma_{0}=\Delta \cup\left\{\square\left(R^{+} x \& H \neg R^{+} x\right)\right\}$

2) Let $\delta_{0} \ldots \delta_{n} \ldots$ be an enumeration of the wffs of the form $\exists y S \varphi, * \varphi$, or $\exists S \varphi$ . Then:

- if $\delta_{n}$ is of the form $\exists y S \varphi, \Gamma_{n+1}=\Gamma_{n} \cup\left\{\left(\exists y S \varphi \rightarrow\left(\exists y S\left(y={ }_{S} x\right) \& \varphi^{x} / y\right)\right)\right\}$ (where $x$ is the first individual variable new to $\Gamma_{n} \cup\left\{\delta_{n}\right\}$ ),

Australasian Journal of Logic (12:1) 2014, Article no. 5 
- if $\delta_{n}$ is of the form $* \varphi$, then

$$
\Gamma_{n+1}=\left\{\begin{array}{l}
\Gamma_{n} \cup\left\{* \varphi \rightarrow *\left(\square(R x \& H \neg R x) \& \exists y S\left(z==_{S} y\right) \& \sigma^{z} / y\right)\right\}, \text { if } \varphi \text { is } \\
\exists y S \sigma, \text { for some wff } \sigma ; \text { or } \\
\Gamma_{n} \cup\left\{* \varphi \rightarrow *\left(\square(R x \& H \neg R x) \& \exists K(K=L) \& \sigma^{L} / M\right)\right\}, \text { if } \varphi \text { is } \\
\exists M \sigma, \text { for some wff } \sigma ; \\
\text { otherwise } \Gamma_{n} \cup\{* \varphi \rightarrow *(\square(R x \& H \neg R x) \& \varphi)\}
\end{array}\right.
$$

(where (a) both $R$ is the first predicate variable and $x$ first individual variable new to $\Gamma_{n} \cup\left\{\delta_{n}\right\}$, if $\varphi$ is not of the form $\exists y S \sigma$ or $\exists S \sigma$ for some wff $\sigma$ and,(b) if $\varphi$ is $\exists y S \sigma$ for some wff $\sigma, z$ is the first individual variable new to $\Gamma_{n} \cup\left\{\delta_{n}\right\}$ ), and both $R$ is the first predicate variable and $x$ the first individual variable new to $\Gamma_{n} \cup\left\{* \varphi \rightarrow *\left(\exists y S\left(z={ }_{S} y\right) \& \sigma^{z} / y\right)\right\}$; (c)if $\varphi$ is $\exists M \sigma$ for some wff $\sigma, L$ is the first sortal term variable new to $\left.\Gamma_{n} \cup\left\{\delta_{n}\right\}\right)$, and both $R$ is the first predicate variable and $x$ is the first individual variable new to $\Gamma_{n} \cup\left\{* \varphi \rightarrow *\left(\exists M\left(L={ }_{M} M\right) \& \sigma^{L} / M\right)\right\}$

- If $\delta_{n}$ is of the form $\exists M \varphi$,

$$
\Gamma_{n+1}=\Gamma_{n} \cup\left\{\exists M \varphi \rightarrow\left(\exists M\left(L={ }_{M} M\right) \& \varphi^{L} / M\right)\right\}
$$

(where $L$ is the first sortal term variable new to $\Gamma_{n} \cup\left\{\delta_{n}\right\}$ ).

By weak induction, it can be shown that $\Gamma_{n}$ is consistent, for every $n \in \omega$. Set $\Gamma^{*}={ }_{n \in \omega} \Gamma_{n}$. Clearly, $\Gamma^{*}$ is consistent. By Lindenbaum's method, extend $\Gamma^{*}$ to a maximally consistent set $\Delta^{*}$. Note that by construction $\Delta^{*}$ is $\omega$-complete and irreflexive. So $\Delta^{*}$ is maxc.

Define now a relation among the set of individual variables as follows:

$x \cong z$ if and only if either for some sortal term variable $M, F \diamond\left(x={ }_{M} z\right) \in \Delta^{*}$ or $P \diamond\left(x={ }_{M} z\right) \in \Delta^{*}$ or $\diamond\left(x={ }_{M} z\right) \in \Delta^{*}$; or for every sortal term variable $M$, $[t] \square\left(\left(\neg \exists y M\left(y={ }_{M} x\right) \& \neg \exists y M\left(y={ }_{M} z\right)\right) \in \Delta^{*}\right.$.

Statement 0: $\cong$ is an equivalence relation in the set of individual variables Proof:

$1) \cong$ is reflexive, i.e., $x \cong x$, for every individual variable $x$ : by Reductio Ad Absurdum, PL, T5,T14, T18 and T19 .

$2) \cong$ is symmetric, that is, if $x \cong z$, then $z \cong x$, for every individual variable $z$ and $x$ : by T9, T10, T11, T30 and PL.

$3) \cong$ is transitive, i.e., if $x \cong z$ and $z \cong w$, then $x \cong w$, for every individual variable $x, z$ and $w$ : by PL, T17, T20-T25, T27-8, T29, T14-16 and consistency of $\Delta^{*}$.

Let $[x]$ be the equivalence class of $x$ determined by $\cong$ in the set of individual variables and set $\mathcal{D}=\{[x] \mid x$ is an individual variable $\}$.Define now a relation among the equivalence classes of $\operatorname{maxc}$ sets of wffs modulo $\simeq_{G}$ as follows: $[\Gamma]_{\simeq_{G}} \equiv$ $\left[\Gamma^{\prime}\right]_{{ }_{G}}$ if and only if there are $\Sigma, \Sigma^{\prime}$ such that $\Sigma \simeq_{G} \Gamma$ and $\Sigma^{\prime} \simeq_{G} \Gamma^{\prime}$ and $\Sigma R_{\square} \Sigma^{\prime}$.

Statement $1: \equiv$ is an equivalence relation.

Proof: Clearly, $\equiv$ is symmetric and reflexive. By A23-24, lemma 10, the irreflexivity of maxc sets, T31, lemma 4 and the fact that $R_{\square}$ is an equivalence relation, it can be shown that $\equiv$ is transitive. 
Set $\sum=\left\{[\Gamma]_{\simeq_{G}} \mid[\Gamma]_{\simeq_{G}} \equiv\left[\Delta^{*}\right]_{\simeq_{G}}\right\}$. It is clear that every equivalence class modulo $R_{\square}$ or modulo $\simeq_{G}$ is a subset $\sum$ or is disjoint with $\sum$.

Statement 2: If $A$ is an equivalence class modulo $\simeq_{G}$ and $B$ an equivalence class modulo $R_{\square}$ both of which are subsets of $\sum$, then there is exactly one maxc $\Xi$ of wffs such that $\Xi \in A \cap B$.

Proof: Assume hypothesis. Then for some $\Phi, \Psi \in \sum,[\Phi]_{\simeq_{G}}=A$ and $[\Psi]_{R_{\square}}=$ $B,[\Phi]_{\simeq_{G}} \equiv\left[\Delta^{*}\right]_{\simeq_{G}} ;$ also, $[\Psi]_{\simeq_{G}} \equiv\left[\Delta^{*}\right]_{\simeq_{G}}$ (since $\Psi \in \sum$ ). By statement $1, \equiv$ is symmetric and transitive, and so $[\Phi]_{\simeq_{G}} \equiv[\Psi]_{\simeq_{G}}$. Also, by the irreflexivity of $\Psi$, A12 and lemma $4,[\Psi]_{R_{\square}} \cap[\Psi]_{\simeq_{G}}=\Psi$. By A23-24, lemma 10, the irreflexivity of maxc sets, T31, lemma 4 , there is a unique maxc $\Xi \in[\Phi]_{\sim_{G}}$ such that $\Psi R_{\square} \Xi$, i.e., $\Xi \in[\Psi]_{R_{\square}}$. Consequently there is a unique maxc $\Xi$ such that $\Xi \in[\Phi]_{\sim_{G}} \cap[\Psi]_{R_{\square}}$.

Convention 5: Given Statement 2, if $A$ is an equivalence class modulo $\simeq_{G}$ and $B$ an equivalence class modulo $R_{\square}$ both of which are subsets of $\sum$, we shall denote the unique $\Gamma \in A \cap B$ by the expression $\Sigma_{(A, B)}^{*}$.

Set $\mathcal{T}=\left\{[\Gamma]_{R_{\square}} \mid[\Gamma]_{R_{\square}} \subseteq \sum\right\}$ and $\mathcal{W}=\left\{[\Gamma]_{\simeq_{G}} \mid[\Gamma]_{\simeq_{G}} \subseteq \sum\right\}$ and

- for every sortal term variable $M$, let $C_{M}$ be the set of ordered pairs $\langle a, b\rangle$ such that:

1) $a \in \mathcal{W} \times \mathcal{T}$ and, where $a$ is $\left([\Gamma]_{\simeq_{G}},[\Theta]_{R_{\square}}\right)$,

$2) b=\left\{[x] \in \mathcal{D} \mid \exists y M\left(x={ }_{M} y\right) \in \Sigma_{\left([\Gamma]_{\simeq_{G}},[\Theta]_{R_{\square}}\right)}^{*}\right\}$

In other words,

$C_{M}=\left\{<\left(\left([\Gamma]_{\simeq_{G}},[\Theta]_{R_{\square}}\right)\right),\left\{[x] \in \mathcal{D} \mid \exists y M\left(x==_{M} y\right) \in \Sigma_{\left([\Gamma]_{\simeq_{G}}^{*},[\Theta]_{R_{\square}}\right)}^{*}\right\}>\right.$ $\mid\left([\Gamma]_{\simeq_{G}},[\Theta]_{R_{\square}}\right) \in \mathcal{W} \times \mathcal{T}$ and $y$ is a variable other than $\left.x\right\}$

- let $\mathcal{S}$ be the set of ordered pairs $\langle a, b\rangle$ such that:

1) $a \in \mathcal{W} \times \mathcal{T}$ and, where $a$ is $\left([\Gamma]_{\simeq_{G}},[\Theta]_{R_{\square}}\right)$,

$2) b=\left\{C_{M} \mid M\right.$ is a sortal term variable such that $\exists L(L=M) \in$ $\Sigma_{\left([\Gamma]_{\simeq_{G}}^{*},[\Theta]_{R_{\square}}\right)}$,provided $L$ is a sortal term variable other than $\left.M\right\}$

In other words,

$\mathcal{S}=\left\{<\left(\left([\Gamma]_{\simeq_{G}},[\Theta]_{R_{\square}}\right),\left\{C_{M} \mid M\right.\right.\right.$ is a sortal term variable such that $\exists L(L=M) \in \Sigma_{\left([\Gamma]_{\sim_{G}},[\Theta]_{R_{\square}}\right)}$, provided $L$ is a sortal term variable other than $\left.M\} \mid\left([\Gamma]_{\simeq_{G}},[\Theta]_{R_{\square}}\right) \in \mathcal{W} \times \mathcal{T}\right\}$.

- for every $n$-place predicate variable $\pi, \mathcal{D}_{\pi}=\left\{<<\left([\Gamma]_{\sim_{G}},[\Theta]_{R_{[K]}}\right)>\right.$, $\left\{<\left[x_{1}\right] \ldots\left[x_{n}\right]>\in \mathcal{D}^{n} \mid \pi x_{1} \ldots x_{n} \in \Sigma_{\left([\Gamma]_{\simeq_{G}},[\Theta]_{R_{[K]}}\right)}^{*}\right\}>\mid\left([\Gamma]_{\simeq_{G}},[\Theta]_{R_{[K]}}\right) \in$ $\mathcal{W} \times T\}$

- $\mathcal{R}=\left\{\left([\Theta]_{R_{\square}},[\Psi]_{R_{\square}}\right) \in \mathcal{T} \times \mathcal{T} \mid\right.$ there is a $[\Gamma]_{\simeq_{G}} \in \mathcal{W}$ such that $\left.\Sigma_{\left([\Gamma]_{\simeq_{G}},[\Theta]_{R_{\square}}\right)}^{*} R_{G} \Sigma_{\left([\Gamma]_{\simeq_{G}}^{*},[\Psi]_{R_{\square}}\right)}\right\}$

Australasian Journal of Logic (12:1) 2014, Article no. 5 
- $\mathcal{A}$ be the function whose domain is the set of variables such that $\mathcal{A}(x)=$ $[x], \mathcal{A}(\pi)=\mathcal{D}_{\pi}$ and $\mathcal{A}(M)=C_{M}$.

- $\mathfrak{A}^{*}=<<\mathcal{D}, \mathcal{S}, \mathcal{W}, \mathcal{T}, \mathcal{R}>, \mathcal{A}>$.

Clearly, $\mathfrak{A}^{*}$ is a VMTS-model.

By Lemmas 5-8 and the soundness theorem, the following statements 3 and 4 can be easily shown:

Statement 3: For any wff $\varphi$, individual variables $y$ and $x$, and $\Gamma \in \mathcal{W}, \Theta \in \mathcal{T}$, $\operatorname{Val}\left(\varphi, \mathfrak{A}^{*}([x] / y), \Gamma, \Theta\right)=1$ if and only if $\operatorname{Val}\left(\left[\varphi^{(x)}\right]^{x} / y, \mathfrak{A}^{*}, \Gamma, \Theta\right)=1$.

Statement 4: For any wff $\varphi$, sortal term variables $L$ and $S$ and $\Gamma \in \mathcal{W}, \Theta \in \mathcal{T}$, $\operatorname{Val}\left(\varphi, \mathfrak{A}^{*}\left(C_{L} / S\right), \Gamma, \Theta\right)=1$ if and only if $\operatorname{Val}\left(\left[\varphi^{(L)}\right]^{L} / S, \mathfrak{A}^{*}, \Gamma, \Theta\right)=1$.

Statement 5: For any $[\Theta]_{R_{\square}},[\Psi]_{R_{\square}} \in \mathcal{T}$, and $[\Gamma]_{\simeq_{G}}, \quad[K]_{\simeq_{G}} \in \mathcal{W}$, if $\Sigma_{\left([K]_{\simeq_{G}},[\Theta]_{R_{\square}}\right)}^{*} R_{G} \Sigma_{\left([K]_{\simeq_{G}},[\Psi]_{R_{\square}}\right)}^{*}$, then $\Sigma_{\left([\Gamma]_{\simeq_{G}},[\Theta]_{R_{\square}}\right)} R_{G} \Sigma_{\left([\Gamma]_{\simeq_{G}},[\Psi]_{R_{\square}}\right)}^{*}$.

Proof: Similar to the proof for Statement 4 in Freund (2007) (p. 593).

Let $I=\left\{\iota \in \omega \mid\right.$ if $\varphi$ is of complexity $\iota$, then for every $\Gamma, \Theta \in \operatorname{Val}\left(\varphi, \mathfrak{A}^{*}\right.$, $\left.[\Gamma]_{\simeq_{G}},[\Theta]_{R_{\square}}\right)=1$ iff $\left.\varphi \in \Sigma_{\left([\Gamma]_{\simeq_{G}},[\Theta]_{R_{\square}}\right)}^{*}\right\}$. By strong induction, it can be shown that $\omega \subseteq I$ (see Proposition II in the Appendix). Therefore, for every wff $\varphi$ and $\Gamma, \Theta \in \operatorname{Val}\left(\varphi, \mathfrak{A}^{*},[\Gamma]_{\simeq_{G}},[\Theta]_{R_{\square}}\right)=1$ iff $\varphi \in \Sigma_{\left([\Gamma]_{\sim_{G}},[\Theta]_{R_{\square}}\right)}$, in particular, for every wff $\varphi, \operatorname{Val}\left(\varphi, \mathfrak{A}^{*},\left[\Delta^{*}\right]_{\simeq_{G}},\left[\Delta^{*}\right]_{R_{\square}}\right)=1$ iff $\varphi \in \Sigma_{\left(\left[\Delta^{*}\right]_{\simeq_{G}},\left[\Delta^{*}\right]_{R_{\square}}\right)}$, since $\left[\Delta^{*}\right]_{\simeq_{G}} \in \mathcal{W}$ and $\left[\Delta^{*}\right]_{R_{\square}} \in \mathcal{T}$. By Statement 2 and the fact that both $\Delta^{*} \in$ $\left[\Delta^{*}\right]_{\simeq_{G}}$ and $\Delta^{*} \in\left[\Delta^{*}\right]_{R_{\square}}, \Delta^{*}=\Sigma_{\left(\left[\Delta^{*}\right]_{\mathcal{G}_{G}},\left[\Delta^{*}\right]_{R_{\square}}\right)}$; but by construction $\Delta \subseteq$ $\Delta^{*}$, and consequently $\operatorname{Val}\left(\psi, \mathfrak{A}^{*},\left[\Delta^{*}\right]_{\simeq_{G}},\left[\Delta^{*}\right]_{R_{\square}}\right)=1$, for every $\psi \in \Delta$, which proves the metatheorem. 


\section{Notes}

${ }^{1}$ Examples of common nouns that are not considered to be sortal terms by many authors are words such as "thing" and "object". For a detailed discussion of these and other related topics see, for example, Geach(1980), Gallois(1988), Deutsch(2007), McGinn(2000), Noonan(1999) and Wiggins(2001). For a complete presentation of the different criteria proposed in the literature for a term to be considered a sortal and the issues regarding those criteria, see Grandy (2007).

${ }^{2}$ Criteria of individuation or identity is not here understood in the sense of a principle of metaphysical individuation, but rather as criteria by means of which we can identify an entity as an individual. Criteria of the latter sort would provide principles for identifying the same object in different contexts, such as time, space, possible worlds, etc. Thus, sortals terms as understood in this paper will provide criteria by means of which we can distinguish something as an individual but not necessarily a metaphysical principle responsible for the individuation of such an individual.

${ }^{3}$ Sortal predication has been shown to be definable in terms of both sortal quantification and sortal identity as follows: $x$ is an $A==_{\text {def }}(\exists y A)\left(y={ }_{A} x\right)$. For this reason were are not including it in our definition of a language for sortals.

${ }^{4}$ See Freund (2007),(2004),(2002) and (2001).

${ }^{5}$ For different versions of nominalism and their explanations of attribution see Rodriguez-Pereyra (2011), (2002), Armstrong (1989)(1989b). For realism, see Armstrong (1989). See Loux and Zimmerman (2003) for philosophical discussions related to both philosophical theories. In the case of sortal predicates, for a realist logical theory see Gupta (1980).

${ }^{6}$ See Cocchiarella (2007) and (1986) for further details on this modern variant of conceptualism.

${ }^{7}$ In the modern variant of conceptualism assumed in this paper, sentences are given a different treatment. For example, assertions are viewed as the expression of the joint exercise of referential concepts and predicable concepts.

${ }^{8}$ Several strong arguments in favor of Leibniz's law for relative identity have been formulated that justify our assuming the law in question for the semantics (see, for example, Wiggins (2001) and Stevenson (1972)). For the different discussions regarding relative identity, see Deutsch (2007). For arguments purporting to show that Leibniz law under sortal or relative identity is untenable, see Geach (1972, pp. 238-47), (1973) and (1980). 


\section{Appendix}

The following propositions and their proofs are instrumental in two of the above proofs. Proposition I is employed in the proof of Lemma 10 and Proposition II at the end of the proof of Metatheorem II (the Completeness Theorem).

Proposition I. If $\varphi, \gamma_{0} \ldots \gamma_{n}$ are wffs and $\Gamma$ is a maxc, then:

(1) If $\odot\left(\gamma_{0} \& \ldots \quad \& \gamma_{n} \& \exists M \varphi\right) \in \Gamma$, then there there is a sortal term variable $L$ other than $M$ which is free for $M$ in $\varphi$ such that $\odot\left(\gamma_{0} \& \ldots \& \gamma_{n} \&\right.$ $\left.\exists M(M=L) \& \varphi^{L} / M\right) \in \Gamma$.

(2) If $\odot\left(\gamma_{0} \& \ldots \& \gamma_{n} \& \exists y S \varphi\right) \in \Gamma$, then there is a individual variable $x$ other than $y$ which is free for $y$ in $\varphi$ such that $\odot\left(\gamma_{0} \& \ldots \& \gamma_{n} \& \exists y S\left(y={ }_{S} x\right)\right.$ $\left.\& \varphi^{x} / y\right) \in \Gamma$

(3) (a) If $\odot\left(\left(\gamma_{0} \& \ldots \& \gamma_{n}\right) \& * \varphi\right) \in \Gamma, \varphi$ is of the form $\exists y S \sigma$, then there is an individual variable $w$ other than $y$ which is free for $y$ in $\sigma$, an one-place predicate variable $R$ which do not occur in $\odot\left(\left(\gamma_{0} \& \ldots \& \gamma_{n}\right) \& *\left(\exists y S\left(y={ }_{S} w\right) \& \sigma^{w} / y\right)\right)$ and an individual variable $x$ such that $\odot\left(\left(\gamma_{0} \& \ldots \& \gamma_{n}\right) \& *(\square(R x \& H \neg R x) \&\right.$ $\left.\left.\exists y S\left(y={ }_{S} w\right) \& \sigma^{w} / y\right)\right) \in \Gamma$

(b) If $\odot\left(\left(\gamma_{0} \& \ldots \& \gamma_{n}\right) \& * \varphi\right) \in \Gamma$ and $\varphi$ is of the form $\exists S \sigma$, then there is sortal term variable $L$ other than $S$ which is free for $S$ in $\sigma$, an one-place predicate variable $R$ which do not occur in $\odot\left(\left(\gamma_{0} \& \ldots \& \gamma_{n}\right) \& *\left(\exists S(S=L) \& \sigma^{L} / S\right)\right)$ and individual variable $x$ such that $\odot\left(\left(\gamma_{0} \& \ldots \quad \& \gamma_{n}\right) \& *(\square(R x \& H \neg R x) \&\right.$ $\left.\left.\exists S(S=L) \& \sigma^{L} / S\right)\right) \in \Gamma$.

(c) If $\odot\left(\left(\gamma_{0} \& \ldots \& \gamma_{n}\right) \& * \varphi\right) \in \Gamma$ and $\varphi$ is neither of the form $\exists y S \sigma$ nor of the form $\exists S \sigma$, there is an one-place predicate variable $R$ which do not occur in $\odot\left(\left(\gamma_{0} \& \ldots \& \gamma_{n}\right) \& * \varphi\right)$ and individual variable $x$ such that $\odot\left(\left(\gamma_{0} \& \ldots\right.\right.$ $\left.\left.\& \gamma_{n}\right) \& *(\square(R x \& H \neg R x) \& \varphi)\right) \in \Gamma$.

Proof:

(1) Suppose $\odot\left(\gamma_{0} \& \ldots \& \gamma_{n} \& \exists M \varphi\right) \in \Gamma$ and let $D$ be a variable new to $\gamma_{0}, \ldots, \gamma_{n}, \exists M \varphi$. Then by PL, $U G(s), \mathrm{A} 9, \mathrm{~A} 4, \mathrm{~T} 2, \mathrm{R} \odot$, Dist $\square$ and definitions, $\odot \exists D\left(\gamma_{0} \& \ldots \& \gamma_{n} \& \varphi^{D} / M\right) \in \Gamma$. Since $\Gamma$ is $\omega$-complete, there is a sortal term variable $L$ other than $D$ which is free for $D$ in $\varphi^{D} / M$ such that $\odot\left(\gamma_{0} \& \ldots\right.$ \& $\left.\gamma_{n} \& \exists D(D=L) \& \varphi^{D} / M^{L} / D\right) \in \Gamma$. Since $D$ is new to $\exists M \varphi$, then $\varphi^{D} / M^{L} / D$ is $\varphi^{L} / M$

(2) Suppose $\odot\left(\gamma_{0} \& \ldots \& \gamma_{n} \& \exists y S \varphi\right) \in \Gamma$ and let $z$ be a variable new to $\gamma_{0}, \ldots, \gamma_{n}, \exists y S \varphi$. Then by UG, PL, A8, A3, T1, R๑, Dist $\square$ and definitions, $\odot \exists z S\left(\gamma_{0} \& \ldots \quad \& \gamma_{n} \& \varphi^{z} / y\right) \in \Gamma$. But $\Gamma$ is $\omega$-complete and so there is a individual variable $x$ other than $z$ which is free for $z$ in $\varphi^{z} / y$ such that $\odot\left(\gamma_{0} \&\right.$ $\left.\ldots \& \gamma_{n} \& \exists z S\left(z={ }_{S} x\right) \& \varphi^{z} / y^{x} / z\right) \in \Gamma$. Since $z$ is new to $\exists y S \varphi$, then " $\varphi^{z} / y^{x} / z$ " is " $\varphi^{x} / y$ ".

(3) (a) If $\odot\left(\left(\gamma_{0} \& \ldots \& \gamma_{n}\right) \& * \varphi\right) \in \Gamma$ and $\varphi$ is of the form $\exists y S \sigma$, then by the $\omega$-completeness of $\Gamma$ there is a individual variable $w$ other than $y$ which is free for $y$ in $\sigma$ such that $\odot\left(\left(\gamma_{0} \& \ldots \& \gamma_{n}\right) \& *\left(\exists y S\left(y={ }_{S} w\right) \& \sigma^{w} / y\right)\right) \in \Gamma$. It follows, by the irreflexivity of $\Gamma$, that there is an one-place predicate variable $R$ which do not occur in $\odot\left(\left(\gamma_{0} \& \ldots \& \gamma_{n}\right) \& *\left(\exists y S\left(y={ }_{S} w\right) \& \sigma^{w} / y\right)\right)$ and individual variable $x$ such that $\odot\left(\left(\gamma_{0} \& \ldots \& \gamma_{n}\right) \& *\left(\square(R x \& H \neg R x) \& \exists y S\left(y={ }_{S} w\right) \&\right.\right.$ 
$\left.\left.\sigma^{w} / y\right)\right) \in \Gamma$.(b) If $\odot\left(\left(\gamma_{0} \& \ldots \& \gamma_{n}\right) \& * \varphi\right) \in \Gamma$ and $\varphi$ is of the form $\exists S \sigma$,then by the $\omega$-completeness of $\Gamma$ there is a individual variable $L$ other than $S$ which is free for $S$ in $\sigma$ such that $\odot\left(\left(\gamma_{0} \& \ldots \& \gamma_{n}\right) \& *\left(\exists S(S=L) \& \sigma^{L} / S\right)\right) \in \Gamma$. It follows, by the irreflexivity of $\Gamma$, that there is an one-place predicate variable $R$ which do not occur in $\odot\left(\left(\gamma_{0} \& \ldots \& \gamma_{n}\right) \& *\left(\exists S(S=L) \& \sigma^{L} / S\right)\right)$ and individual variable $x$ such that $\odot\left(\left(\gamma_{0} \& \ldots \quad \& \gamma_{n}\right) \& *(\square(R x \& H \neg R x) \& \exists S(S=L) \&\right.$ $\left.\left.\sigma^{L} / S\right)\right) \in \Gamma$. Finallly, (c) If $\odot\left(\left(\gamma_{0} \& \ldots \& \gamma_{n}\right) \& * \varphi\right) \in \Gamma$ and $\varphi$ is neither of the form $\exists y S \sigma$ nor of the form $\exists S \sigma$, then by the irreflexivity of $\Gamma$ there is an one-place predicate variable $R$ which do not occur in $\odot\left(\left(\gamma_{0} \& \ldots \& \gamma_{n}\right) \&\right.$ $* \varphi)$ and individual variable $x$ such that $\odot\left(\left(\gamma_{0} \& \ldots \& \gamma_{n}\right) \& *(\square(R x \& H \neg R x) \&\right.$ $\varphi)) \in \Gamma$

Proposition II. If $I=\{\iota \in \omega \mid$ if $\varphi$ is of complexity $\iota$, then for every $\Gamma, \Theta \in$, $\operatorname{Val}\left(\varphi, \mathfrak{A}^{*},[\Gamma]_{\simeq_{G}},[\Theta]_{R_{\square}}\right)=1$ iff $\left.\varphi \in \Sigma_{\left([\Gamma]_{\simeq_{G}},[\Theta]_{R_{\square}}\right)}^{*}\right\}$, then $\omega \subseteq I$.

Proof: suppose that $\varphi$ is of complexity $k, \Gamma, \Theta \in \sum$ and for every $i<k$, $i \in I$. There are seven cases to consider. The cases where $\varphi$ is of the form $\neg \gamma$ or $\gamma \rightarrow \sigma$ can be easily shown by the inductive hypothesis. We omit proofs for the cases where $\varphi$ is of the form $\pi x_{1} \ldots x_{n}, \forall y M \gamma, G \varphi, H \varphi$ or $\square \varphi$ because they are similar to the proofs of those cases in Freund (2007) (p. 595).

1. $\varphi$ is of the form $x=_{M} y: \operatorname{Val}\left(\varphi, \mathfrak{A}^{*},[\Gamma]_{\simeq_{G}},[\Theta]_{R_{\square}}\right)=1$ if and only if (by definition) $\mathcal{A}(x)=\mathcal{A}(y)$ and $\mathcal{A}(y) \in \mathcal{A}(M)\left([\Gamma]_{\widetilde{ }_{G}},[\Theta]_{R_{\square}}\right)$ if and only if (by definition) $[x]=[y]$ and $\exists z M\left(z={ }_{M} y\right) \in \Sigma_{\left([\Gamma]_{\beth_{G}},[\Theta]_{R_{\square}}\right)}$ if and only if (by definition) either for some sortal term variable $B, P \diamond\left(x={ }_{B} y\right) \in \Delta^{*}$ or $F \diamond\left(x={ }_{B} y\right) \in \Delta^{*}$ or $\diamond\left(x={ }_{B} y\right) \in \Delta^{*}$; or for every sortal term variable $B$, $[t] \square\left(\neg \exists z B\left(z={ }_{B}\right.\right.$ $\left.x) \& \neg \exists z B\left(z={ }_{B} y\right)\right) \in \Delta^{*}$, and $\exists z M\left(z={ }_{M} y\right) \in \Sigma_{\left([\Gamma]_{\sim_{G}},[\Theta]_{R_{\square}}\right)}^{*}$.

Suppose first that for some sortal term variable $B, F \diamond\left(x={ }_{B} y\right) \in \Delta^{*}$ or $P \diamond\left(x={ }_{B} y\right) \in \Delta^{*}$ or $\diamond\left(x==_{B} y\right) \in \Delta^{*}$, and $\exists z M\left(z={ }_{M} y\right) \in \Sigma_{\left([\Gamma]_{\simeq_{G}},[\Theta]_{R_{\square}}\right)}^{*}$. By A10, PL and the fact that $R_{G}$ is a serial ordering in $\left[\Delta^{*}\right]_{\simeq_{G}}, \square\left(\left(\exists x M\left(y={ }_{M}\right.\right.\right.$ $\left.x) \rightarrow\left(y==_{M} z\right)\right) \in \Sigma_{\left(\left[\Delta^{*}\right]_{\simeq_{G}},[\Theta]_{R_{\square}}\right)}$. But $\Sigma_{\left(\left[\Delta^{*}\right]_{\sim_{G}},[\Theta]_{R_{\square}}\right)}^{*} \in[\Theta]_{R_{\square}}$ and so $\square\left(\exists x M\left(y={ }_{M} x\right) \rightarrow\left(y={ }_{M} z\right) \in \Sigma_{\left([\Gamma]_{\sim_{G}}^{*},[\Theta]_{R_{\square}}\right)}\right.$, from which it follows by A12 that $\left(\exists x M\left(y==_{M} x\right) \rightarrow\left(y==_{M} z\right)\right) \in \Sigma_{\left([\Gamma]_{\sim_{G}},[\Theta]_{R_{\square}}\right)}$. But given that $\exists z M\left(z={ }_{M} y\right) \in \Sigma_{\left([\Gamma]_{\sim_{G}},[\Theta]_{R_{\square}}\right)}^{*}$, by PL $\left.\left(y={ }_{M} z\right)\right) \in \Sigma_{\left([\Gamma]_{\simeq_{G}},[\Theta]_{R_{\square}}\right.}^{*}$.

On the other hand, if for every sortal term variable $M,[t] \square\left(\neg \exists z M\left(x={ }_{M} z\right)\right.$ $\left.\& \neg \exists z M\left(y==_{M} z\right)\right) \in \Delta^{*}$, then $\square\left(\neg \exists z M\left(x={ }_{M} z\right) \& \neg \exists z M\left(y={ }_{M} z\right)\right) \in$ $\Sigma_{\left(\left[\Delta^{*}\right]_{\beth_{G}},[\Theta]_{R_{\square}}\right)}$, since $R_{G}$ is a serial ordering in $\left[\Delta^{*}\right]_{\simeq_{G}}$. Clearly, since $\Sigma_{\left(\left[\Delta^{*}\right]_{\sim_{G}},[\Theta]_{R_{\square}}\right)}^{*} \in[\Theta]_{R_{\square}}, \square\left(\neg \exists z M\left(x==_{M} z\right) \& \neg \exists z M\left(y==_{M} z\right)\right) \in \Sigma_{\left([\Gamma]_{\sim_{G}},[\Theta]_{R_{\square}}\right)}^{*}$ and by $\mathrm{A} 12\left(\neg \exists z M\left(x=_{M} z\right) \& \neg \exists z M\left(y==_{M} z\right)\right) \in \Sigma_{\left([\Gamma]_{\sim_{G}}^{*},[\Theta]_{R_{\square}}\right)}$, which is impossible because by assumption $\exists z M(y=M z) \in \Sigma_{\left([\Gamma]_{\sim_{G}},[\Theta]_{R_{\square}}\right)}^{*}$. Therefore, it is not the case that for every sortal term variable $M$, $[t] \square\left(\neg \exists z M\left(x={ }_{M} z\right)\right.$ $\left.\& \neg \exists z M\left(y={ }_{M} z\right)\right) \in \Delta^{*}$.

Assume now that $x={ }_{M} y \in \Sigma_{\left([\Gamma]_{\simeq_{G}},[\Theta]_{R_{\square}}\right)}$. Then by T4 $\exists z M\left(z={ }_{M}\right.$ $y) \in \Sigma_{\left([\Gamma]_{\sim_{G}},[\Theta]_{R_{\square}}\right)}^{*}$ and by A12 and A13 $\square \diamond\left(x==_{M} y\right) \in \Sigma_{\left([\Gamma]_{\sim_{G}},[\Theta]_{R_{\square}}\right)}^{*}$. 
But $\Sigma_{\left([\Gamma]_{\simeq_{G}}^{*},[\Theta]_{R_{\square}}\right)} \in[\Theta]_{R_{\square}}$ and so $\square \diamond\left(x==_{M} y\right) \in \Sigma_{\left(\left[\Delta^{*}\right]_{\simeq_{G}},[\Theta]_{R_{\square}}\right)}^{*}$, which by A12 means that $\diamond\left(x=_{M} y\right) \in \Sigma_{\left(\left[\Delta^{*}\right]_{\sim_{G}},[\Theta]_{R_{\square}}\right)}^{*}$. Since $R_{G}$ is a serial ordering in $\left[\Delta^{*}\right]_{\sim_{G}}$ and by lemma $0, F \diamond\left(x==_{M} y\right) \in \Delta^{*}$ or $P \diamond\left(x={ }_{M} y\right) \in \Delta^{*}$ or $\diamond\left(x={ }_{M} y\right) \in \Delta^{*}$. Therefore, for some sortal term $M, F \diamond\left(x={ }_{M} y\right) \in \Delta^{*}$ or $P \diamond\left(x==_{M} y\right) \in \Delta^{*}$ or $\diamond\left(x={ }_{M} y\right) \in \Delta^{*}$ and consequently, either for some sortal term $M, F \diamond\left(x={ }_{M} y\right) \in \Delta^{*}$ or $P \diamond\left(x={ }_{M} y\right) \in \Delta^{*}$ or $\diamond\left(x={ }_{M} y\right) \in \Delta^{*}$,or for every sortal some sortal term $M[t] \square\left(\left(\neg \exists z M\left(x={ }_{M} z\right) \& \neg \exists z M\left(y={ }_{M} z\right)\right)\right) \in \Delta^{*}$; and $\exists z M\left(z={ }_{M} y\right) \in \Sigma_{\left([\Gamma]_{\simeq_{G}}^{*},[\Theta]_{R_{\square}}\right)}^{*}$

2. $\varphi$ is of the form $\forall S \gamma: \operatorname{Val}\left(\varphi, \mathfrak{A}^{*},[\Gamma]_{\simeq_{G}},[\Theta]_{R_{\square}}\right)=1$ if and only if(by definition) for every $C_{F} \in \mathcal{S}\left([\Gamma]_{\simeq_{G}},[\Theta]_{R_{\square}}\right), \operatorname{Val}\left(\gamma, \mathfrak{A}^{*}\left(C_{F} / S\right),[\Gamma]_{\simeq_{G}},[\Theta]_{R_{\square}}\right)=1$ if and only if (by Statement 4) for every $C_{F} \in \mathcal{S}\left([\Gamma]_{\simeq_{G}},[\Theta]_{R_{\square}}\right), \operatorname{Val}\left(\left[\gamma^{(F)}\right]^{F} / S, \mathfrak{A}^{*}\right.$, $\left.[\Gamma]_{\simeq_{G}},[\Theta]_{R_{\square}}\right)=1$ if and only if (by definition) for every sortal term variable $F$ and individual variables $x$, $y$, if $\exists L(L=S \quad F) \in \Sigma_{\left([\Gamma]_{\sim_{G}}^{*},[\Theta]_{R_{\square}}\right)}$ (where $L$ is a sortal term variable other than $F)$, then $\operatorname{Val}\left(\left[\gamma^{(F)}\right]^{F} / S, \mathfrak{A}^{*},[\Gamma]_{\simeq_{G}},[\Theta]_{R_{\square}}\right)=1$ if and only if (by the inductive hypothesis) for every sortal term variable $F$, if $\exists L\left(L==_{S} \quad F\right) \in \Sigma_{\left([\Gamma]_{\sim_{G}}^{*},[\Theta]_{R_{\square}}\right)}$, then, $\left[\gamma^{(F)}\right]^{F} / S \in \Sigma_{\left([\Gamma]_{\simeq_{G}}^{*},[\Theta]_{R_{\square}}\right)}$ if and only if (by $\omega$-completeness and maximality of $\Sigma_{\left([\Gamma]_{\simeq_{G}}^{*},[\Theta]_{R_{\square}}\right)}$, Lemma 6 and note (ii) inmediately following Lemmas 5-6, A6 and T2) $\forall S \gamma \in \Sigma_{\left([\Gamma]_{\simeq_{G}}^{*},[\Theta]_{R_{\square}}\right)}$.

\section{References}

Armstrong, D.M., 1989, Nominalism and Realism Vol. 1 and 2, Cambridge University Press, Cambridge.

Armstrong, D.M., 1989b, Universals, An Opinionated Introduction, Westview Press, Boulder.

Cocchiarella, Nino, 2007, Formal Ontology and Conceptual Realism, Synthese Library,Vol. 339, Springer Verlag.

Cocchiarella, Nino, 1986, Logical Investigations of Predication Theory and the Problem of Universals, Bibliopolis Press, Naples.

Deutsch, H., 2007,"Relative Identity", in E. Zalta (ed.), Stanford Encyclopedia of Philosophy .

Freund, Max, 2007, "A Two Dimensional Tense-Modal Sortal Logic", Journal of Philosophical Logic, 36, pp. 571-98

Freund, Max, 2004, "A Modal Sortal Logic", Journal of Philosophical Logic, 33, pp. 237-60

Freund, Max, 2002, "A Temporal Logic for Sortals", Studia Logica, 69, pp. 35180

Freund, Max, 2001, "A Complete and Consistent Formal System for Sortals", Studia Logica, 65, pp. 367-81 
Gallois, A., 1998, Occasions of Identity: A Study in the Metaphysics of Persistence, Change and Sameness, Clarendon Press, Oxford.

Geach, Peter, 1972, Logic Matters, University of California Press, Los Angeles. Geach, Peter, 1973, "Ontological relativity and relative identity", in Munitz, M.K. (ed.), Logic and Ontology, NYU Press, New York.

Geach, Peter, 1980, Reference and Generality, Cornell U. Press, Ithaca

Grandy, Richard, 2007, "Sortals", in E. Zalta, ed., Stanford Encyclopedia of Philosophy, http://plato.stanford.edu/archives/spr2014/entries/sortals

Gupta, A., 1980, Logic of Common Nouns, Yale U. Press, Ithaca

Loux, Michael; Zimmerman, Dean, 2003, The Oxford Handbook of Metaphysics, Oxford University Press, New York.

Lovibond, S., and Williams, S.G., 1996, Identity, Truth and Value: Essays for David Wiggins, Aristotelian Society Series, Vol. 16, Blackwell, Oxford.

McGinn, C., 2000, Logical Properties, Blackwell, Oxford.

Noonan, H., 1999, "Relative Identity". In Hale, B. and Wright C.(eds.), A Companion to the Philosophy of Language, Blackwell, Oxford.

Rodriguez-Pereyra, Gonzalo, 2011,"Nominalism in Metaphysics", in E. Zalta (ed.), Stanford Encyclopedia of Philosophy, http://plato.stanford.edu/archives/ sum2014/entries/nominalism-metaphysics/

Rodriguez-Pereyra, Gonzalo, 2002, Resemblance Nominalism: A Solution to the Problems of Universals, Oxford U.P., New York,

Stevenson, L., 1972, "Relative Identity and Leibniz' Law", The Philosophical Quarterly, 22, 155-158.

Wiggins, David, 2001, Sameness and Substance Renewed, Harvard U. Press, Cambridge, MA. 